\title{
Sparse regressions for predicting and interpreting subcellular localization of multi-label proteins
}

\author{
Shibiao Wan ${ }^{1 *}$ (D, Man-Wai Mak ${ }^{1 *}$ and Sun-Yuan Kung ${ }^{2}$
}

\begin{abstract}
Background: Predicting protein subcellular localization is indispensable for inferring protein functions. Recent studies have been focusing on predicting not only single-location proteins, but also multi-location proteins. Almost all of the high performing predictors proposed recently use gene ontology (GO) terms to construct feature vectors for classification. Despite their high performance, their prediction decisions are difficult to interpret because of the large number of $\mathrm{GO}$ terms involved.

Results: This paper proposes using sparse regressions to exploit GO information for both predicting and interpreting subcellular localization of single- and multi-location proteins. Specifically, we compared two multi-label sparse regression algorithms, namely multi-label LASSO (mLASSO) and multi-label elastic net (mEN), for large-scale predictions of protein subcellular localization. Both algorithms can yield sparse and interpretable solutions. By using the one-vs-rest strategy, mLASSO and mEN identified 87 and 429 out of more than 8,000 GO terms, respectively, which play essential roles in determining subcellular localization. More interestingly, many of the GO terms selected by mEN are from the biological process and molecular function categories, suggesting that the GO terms of these categories also play vital roles in the prediction. With these essential GO terms, not only where a protein locates can be decided, but also why it resides there can be revealed.

Conclusions: Experimental results show that the output of both $\mathrm{mEN}$ and $\mathrm{mLASSO}$ are interpretable and they perform significantly better than existing state-of-the-art predictors. Moreover, mEN selects more features and performs better than mLASSO on a stringent human benchmark dataset. For readers' convenience, an online server called SpaPredictor for both mLASSO and mEN is available at http://bioinfo.eie.polyu.edu.hk/SpaPredictorServer/.
\end{abstract}

\section{Background}

Within living organisms, proteins need to locate in the right subcellular compartments to perform various biological functions. Mislocalized human proteins are liable to cause numerous human diseases, such as kidney stone [1], Bartter syndrome [2], primary human liver tumors [3], Alzheimer's disease [4], breast cancer [5], pre-eclampsia [6] and minor salivary gland tumors [7]. Knowing where a protein resides within a cell is an indispensable and essential step to uncover its functions and detect drug targets [8]. Traditional wet-lab techniques

\footnotetext{
*Correspondence: shibiao.wan@connect.polyu.hk; enmwmak@polyu.edu.hk

${ }^{1}$ Department of Electronic and Information Engineering, The Hong Kong

Polytechnic University, Hong Kong SAR, China

Full list of author information is available at the end of the article
}

such as cell fractionation, electron microscopy and fluorescent microscopy imaging, are applied to construct high quality localization databases such as the Human Protein Atlas. ${ }^{1}$ However, the processes are laborious, expensive and time-consuming. To tackle tremendous numbers of newly discovered protein sequences generated by largescale sequencing projects, efficient computational methods are required for fast and accurate prediction of protein subcellular localization (PSCL).

Conventionally, PSCL was tackled by sequence-based approaches. This type of approaches includes three categories: (1) sorting-signals based methods [9-11]; (2) amino-acid composition-based methods [12-15]; (3) homology-based methods [16, 17]. Beyond sequence information, knowledge-based approaches have been developed. This type of approaches uses information 
from knowledge databases, such as Gene Ontology (GO $)^{2}$ terms [18-28], PubMed abstracts [29, 30], or Swiss-Prot keywords [31, 32]. Among these methods, GO-based methods were found to be superior in terms of performance [23, 33-35].

Because many studies [36-39] have found the prevalence of multi-location proteins in living organisms, recent studies have been focusing on predicting not only single-location proteins, but also multi-location proteins. Multi-label proteins are found to participate in various metabolic activities in multiple cellular compartments. For example, the glucose transporter GLUT4 is found in both the plasma membrane and the intracellular vesicles of adipocytes [40, 41]; proteins involved in fatty acid $\beta$-oxidation is found in the mitochondria and peroxisome; and antioxidant defense proteins are known to reside in the peroxisome, cytosol and mitochondria [42].

Many state-of-the-art multi-label predictors - such as iLoc-Hum [27], Hum-mPLoc 2.0 [43], mGOASVM [44], HybridGO-Loc [45], R3P-Loc [46], mPLR-Loc [47] and others [48-50] - use GO information as features and apply different multi-label classifiers to tackle the multilabel classification problem. Nevertheless, due to the high dimensionality of GO features, these GO-based predictors often have the following drawbacks:

1. Lack of interpretability. These predictors can only give insights into where the query proteins are located, but cannot provide biological reasons of why they reside there. This is possibly a common problem for most machine-learning based approaches, because it is usually difficult to correlate the statistical characteristics of biological data with biological phenomena. On the other hand, biologists want to know not only the prediction results of query proteins, but also biological features or factors that lead to the prediction results. Therefore, the lack of interpretability may limit the powers and applications of these predictors. As far as we know, there is only one subcellular-location predictor called YLoc [51] that is interpretable. However, YLoc requires heterogeneous biological features such as sorting signals, PROSITE ${ }^{3}$ patterns and GO terms, which are not always available for every protein.

2. Susceptibility to overfitting. The number of extracted GO features from knowledge databases (e.g., GO annotation database ${ }^{4}$ ) is considerably larger than the number of proteins of interest. Most of the existing predictors (except R3P-Loc) construct feature vectors with dimensions as high as several thousand. Among these thousands of features, it is likely that many are irrelevant or redundant, causing the predictors suffer from overfitting
To tackle the problems mentioned above, this paper proposes two sparse and interpretable multi-label predictors, namely $\mathbf{m L A S S O}$ and $\mathbf{m E N}$ for large-scale predictions of both single- and multi-location proteins. Given a query protein sequence, a set of GO terms are retrieved from two newly created compact databases by the procedures described in [46]. The frequencies of $\mathrm{GO}$ occurrences are used to formulate frequency vectors with dimension over 8000. By using a one-vs-rest LASSO-based (least absolute shrinkage and selection operator-based) classifier and an EN-based (elastic net) classifier, 87 and 429 out of these $8,000+$ GO terms were selected, respectively. With the selected $\mathrm{GO}$ terms, the frequency vectors are converted into dimension-reduced feature vectors (87-dim for mLASSO and 429-dim for mEN). Subsequently, the 87-dim (429dim resp.) feature vectors are classified by a multi-label LASSO (EN resp.) classifier. Experimental results based on a stringent human benchmark dataset demonstrate that the two proposed predictors substantially outperform other state-of-the-art predictors. More significantly, based on GO terms selected by either mLASSO or $\mathrm{mEN}$, researchers can decide not only where a protein resides within a cell, but also why it is located there. Moreover, mEN not only selects more GO terms than mLASSO, but also performs better than mLASSO. We have also found that besides cellular-component $\mathrm{GO}$ terms, GO terms from the categories of molecular functions and biological processes also contribute to the final predictions.

\section{Legitimacy of using GO information}

Some researchers may have reservations about the use of GO information for PSCL. In the following, we list the concerns (C1 and C2) and our explanations (E1 and E2) of why these concerns do not cause problems in PSCL.

C1: Because the cellular component GO terms have already been annotated with cellular component categories, GO-based methods can be simply replaced by a lookup table using the cellular component GO terms as the keys and the component categories as the hashed value.

E1: This naive solution is not recommended because not only the cellular component GO terms are relevant to the PSCL, GO terms from the biological processes and molecular functions also play important roles, as demonstrated in [52]. In particular, it has been found [52] that GO terms in biological process and molecular function categories are particularly relevant to nucleus, extracellular space, membrane, mitochondrion, endoplasmic reticulum and Golgi apparatus. In fact, the relationship between $\mathrm{GO}$ terms and PSCL is not a one-to-one mapping and 
recent studies $[34,44]$ have already shown that this naive solution will lead to very poor performance.

C2: Are GO-based methods equivalent to transferring annotations from BLAST [53] homologs?

E2: This concern is explicitly addressed in our previous study [34], which demonstrated that GO-based methods remarkably outperform methods that only use BLAST and homologous transfer (in Table 4 of [34]). Besides, Briesemeister et al. [54] also found that using BLAST alone is not sufficient for reliable prediction.

In fact, as suggested by Chou [55], given a predictor, as long as its inputs are amino acid sequences and its outputs (predictions) are subcellular localizations, the predictions made by the predictor are legitimate; whether the predictor uses GO-based methods or non GO-based methods is not an issue. Some other papers $[56,57]$ also provide strong evidences supporting the legitimacy of using GO information for subcellular localization. In particular, as suggested by [57], the good performance of GO-based methods is due to the fact that the feature vectors in the GO space can better reflect their subcellular locations than those in the Euclidean space or any other simple geometric space.

\section{Results}

\section{Datasets}

A stringent human benchmark dataset [43] was used to evaluate the performance of mLASSO and mEN. The human dataset was created from Swiss-Prot 55.3, which is a publicly accessible protein database. ${ }^{5}$ This benchmark dataset is downloadable from the hyperlink in the SpaPredictor server. It contains 3106 human proteins distributed in 14 locations. The sequence identity of the dataset was cut off at $25 \%$. Figure 1(a) shows the breakdown of the human dataset. Here, 3106 actual proteins [44] correspond to 3681 locative proteins [44, 58]. ${ }^{6}$ As can be seen from Fig. 1(a), the majority (79.9\%) of the human proteins are located in cytoplasm, nucleus, extracellular, mitochondrion and plasma membrane, while proteins located in the other 9 subcellular locations account for around $20 \%$. This means that the dataset is very imbalanced.

Figure 1(b) further shows the distribution of co-located proteins. As can be seen, of the 3106 proteins, 2580 belong to one subcellular location, 480 belong to two locations, 43 belong to three locations, 3 belong to four locations and none to five or more locations. As shown in the pie charts in Fig. 1(b), the majority of single-location proteins are located in cytoplasm, extracellular, mitochondrion, nucleus and plasma membrane, which is consistent with the distribution of the overall locative proteins shown in Fig. 1(a). For the proteins locating at two subcellular locations, around two thirds of proteins are distributed in cytoplasm and nucleus; on the contrary, proteins residing in three or four subcellular locations are more evenly distributed than those single-location and two-location proteins. This analysis suggests that single-location proteins play more significant roles in shaping the overall distribution of the dataset; however, multi-location proteins also constitute a considerable percentage of the dataset.

\section{Performance metrics}

To facilitate performance comparisons in multi-label classification, some sophisticated performance metrics are introduced here to better reflect the multi-label capabilities of classifiers. These measures include Accuracy, Precision, Recall, F1-score (F1) and Hamming Loss (HL). The definitions of these five measurements for multilabel classification can be found in [45, 46]. Accuracy, Precision, Recall and $F 1$ indicate the classification performance. The higher the measures, the better the prediction performance. Among them, Accuracy is the most commonly used criteria. F1-score is the harmonic mean of Precision and Recall, which allows us to compare the performance of classification systems by taking the trade-off between Precision and Recall into account. The Hamming Loss (HL) $[59,60]$ is different from other metrics in that the former concerns about the misclassified instance-label pairs whereas the latter are more interested in the correctly classified instancelabel pairs. The lower the $H L$, the better the prediction performance. More details about the performance metrics can be found in Section S7 of supplementary materials.

Since partial matching has been widely used in measuring classification performance $[61,62]$, especially in multi-label classification [63], we have also used another two measures: Micro F-measure (Micro F1) and Macro F-measure (Macro F1). The definitions of these two measures in multi-label learning scenarios can be found in [63]. To compute Macro F1, the F1 of individual classes are independently computed and then averaged. As a result, Macro F1 treats the F1 of individual classes equally important and the measure is insensitive to the imbalance in class sizes. On the other hand, to compute Micro F1, the true-positive, true-negative, and false-negative are accumulated across all classes, followed by plugging these values into the standard formula for computing F1. Therefore, Macro F1 considers every binary decision equally important, whereas Micro F1 will be heavily dependent on the decisions (both correct and incorrect) on the large classes [64].

Two additional measures $[44,65]$ are often used in multi-label subcellular localization prediction. They are overall locative accuracy $(O L A)$ and overall actual accuracy $(O A A)$. Specifically, denote $\mathcal{L}\left(\mathbb{Q}_{i}\right)$ and $\mathcal{M}\left(\mathbb{Q}_{i}\right)$ as the 


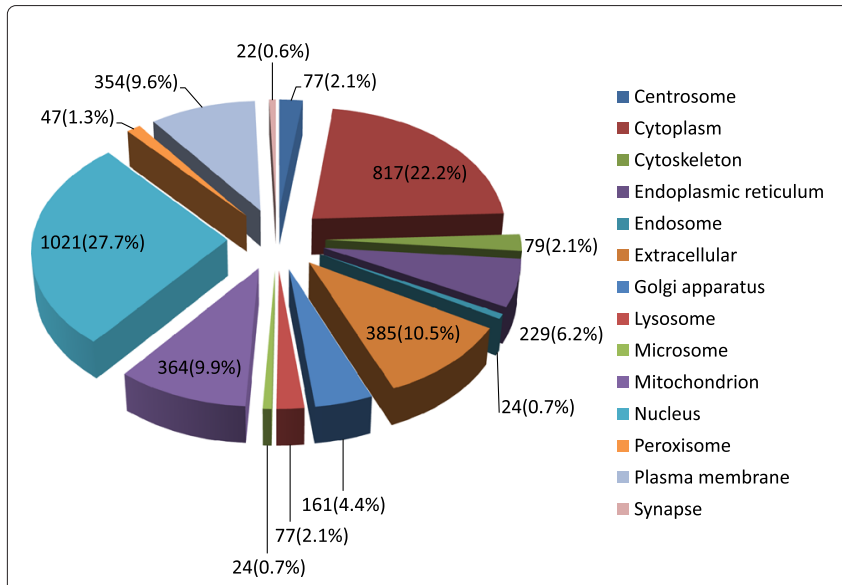

(a) Dataset breakdown

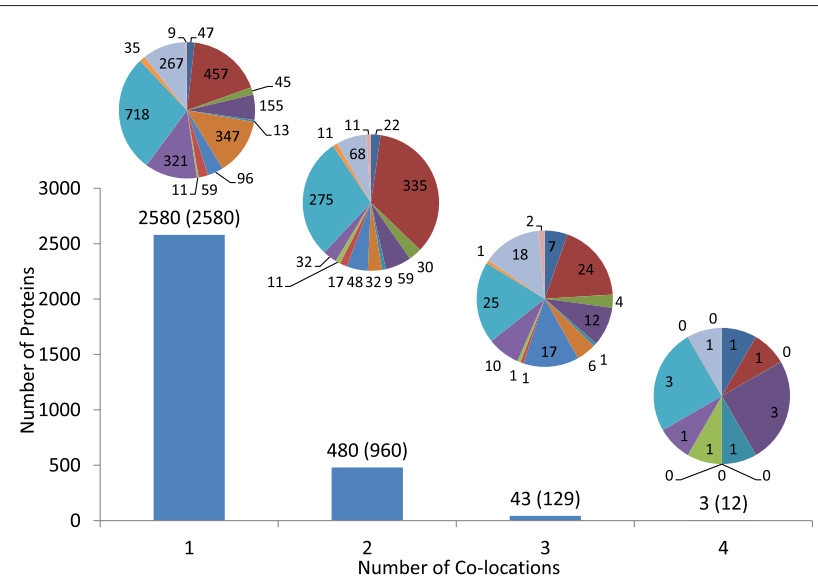

(b) Dataset analysis

Fig. 1 Information of the human dataset. (a) Dataset breakdown; (b) dataset analysis. The number of proteins shown in each subcellular location represents the number of 'locative proteins' $[44,65]$. The dataset comprises 3106 actual proteins and 3681 locative proteins distributed in 14 subcellular locations. In $(\mathbf{b})$, for each bar, the numbers $m(n)$ on top denote that there are $m$ actual proteins and $n$ locative proteins having the same number of co-location(s) indicated in the bottom of the bar. For example, there are 43 actual and 129 locative proteins that have three subcellular locations. The small pie charts show the distribution of locative proteins having the number of co-location(s) shown in the bottom of the bar chart

true label set and the predicted label set for the $i$-th protein $\mathbb{Q}_{i}(i=1, \ldots, N)$, respectively. ${ }^{7}$ Then, $O L A$ is given by:

$$
O L A=\frac{1}{\sum_{i=1}^{N}\left|\mathcal{L}\left(\mathbb{Q}_{i}\right)\right|} \sum_{i=1}^{N}\left|\mathcal{M}\left(\mathbb{Q}_{i}\right) \cap \mathcal{L}\left(\mathbb{Q}_{i}\right)\right|,
$$

and the overall actual accuracy $(O A A)$ is:

$$
O A A=\frac{1}{N} \sum_{i=1}^{N} \Delta\left[\mathcal{M}\left(\mathbb{Q}_{i}\right), \mathcal{L}\left(\mathbb{Q}_{i}\right)\right]
$$

where

$$
\Delta\left[\mathcal{M}\left(\mathbb{Q}_{i}\right), \mathcal{L}\left(\mathbb{Q}_{i}\right)\right]=\left\{\begin{array}{l}
1, \text { if } \mathcal{M}\left(\mathbb{Q}_{i}\right)=\mathcal{L}\left(\mathbb{Q}_{i}\right) \\
0, \text { otherwise }
\end{array}\right.
$$

Among all the metrics mentioned above, $O A A$ is the most stringent and objective. This is because if some (but not all) of the subcellular locations of a query protein are correctly predicted, the numerators of the other five measures (including Accuracy, Precision, Recall, F1 and OLA) are non-zero, whereas the numerator of $O A A$ in Eq. 2 is 0 (thus making no contribution to the frequency count).

Leave-one-out cross validation (LOOCV) is considered to be the most rigorous and bias-free procedure [66] for evaluating classifiers' performance. Hence, LOOCV was used to examine the performance of mLASSO and mEN.

\section{Statistical analysis of the essential GO terms}

Figure 2(a) and (b) show the categorical breakdown of essential GO terms found by mLASSO and mEN. Figure 2(a) shows that for each subcellular location, around 30 50 essential GO terms determine where a protein resides. For example, for cytoplasm, 39 essential GO terms contribute to the final decisions, of which 24 belong to the cellular-component category; the remaining 6 and 9 belong to molecular function and biological process categories, respectively. Besides, around half of the essential GO terms belong to cellular components, e.g., 22 out of 37 in centrosome, 24 out of 39 in cytoplasm, etc. The results indicate that for mLASSO, cellular component GO terms contribute more to the final prediction than the other GO terms. However, as shown in Fig. 2(b), the percentage of cellular-component GO terms found by mEN is much smaller. For example, for cytoplasm, only around $36 \%$ (56 out of 158 ) belongs to cellular components. These results suggest that essential GO terms from biological processes and molecular functions may contribute more to the final predictions for mEN than for mLASSO.

A comparison between Fig. 2(a) and (b) reveals that the number of essential GO terms selected by mEN is much larger than that selected by mLASSO for all of the 14 subcellular locations; this phenomenon also occurs across the three GO categories. This is because GO terms from the same category are not independent on each other; instead they are related in a hierarchical manner. Compared to mLASSO, mEN encourages selecting correlated features together, thus causing more essential GO terms to be selected. The results are consistent with the claims by Zou and Hastie [67].

We used the degree of overlapping among the essential GO terms found by mLASSO and mEN to investigate the relationship between the GO terms found by these algorithms. The results are shown in Fig. 3. Figure 3(a) shows that the 87 essential GO terms selected by mLASSO are 


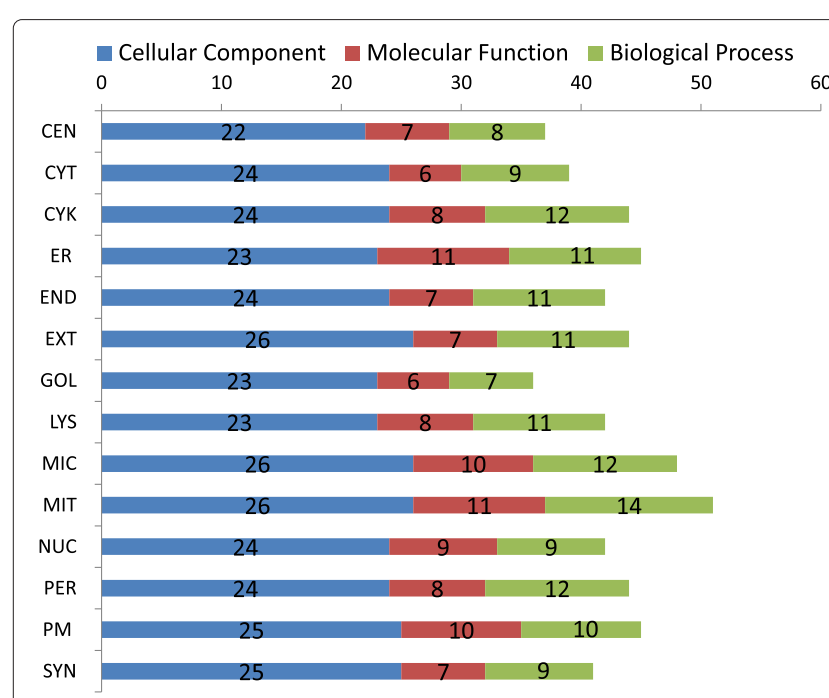

(a) mLASSO

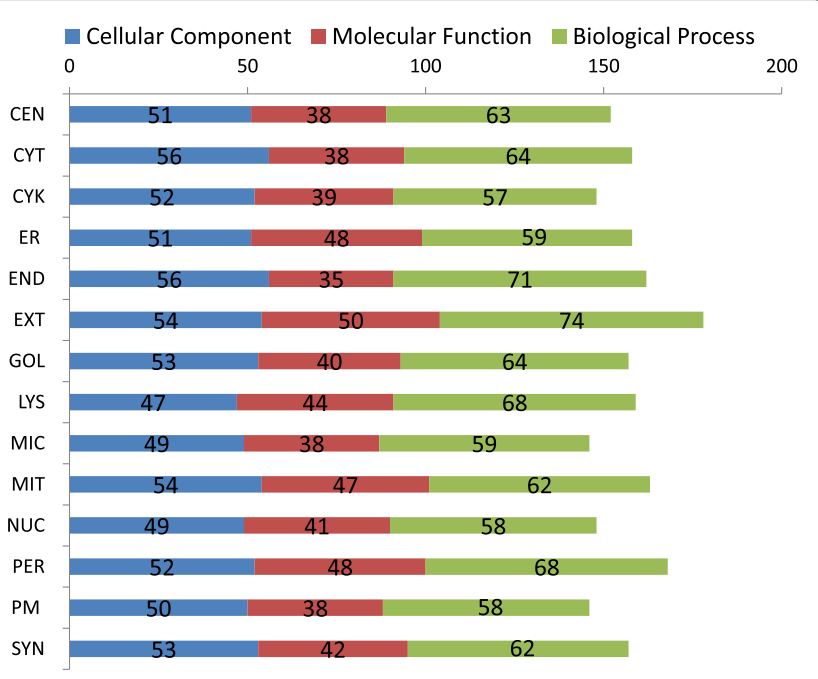

(b) $\mathrm{mEN}$

Fig. 2 The categorical breakdown of the essential GO terms in each subcellular location for (a) mLASSO and (b) mEN. CEN: centrosome; CYT: cytoplasm; CYK: cytoskeleton; ER: endoplasmic reticulum; END: endosome; EXT: extracellular; GOL: Golgi apparatus; LYS: lysosome; MIC: microsome; MIT: mitochondrion; NUC: nucleus; PER: peroxisome; PM: plasma membrane; SYN: synapse

totally included in the 429 essential GO terms selected by $\mathrm{mEN}$. Figure 3(b-d) show that for centrosome, cytoplasm and mitochondrion, there are 36, 38 and 50 overlapped essential GO terms. For each of these subcellular locations, there is only one essential GO term that is found by mLASSO but missed by mEN. Specifically, for centrosome, the GO term missed by $\mathrm{mEN}$ is GO:0005829 (CC, cytosol); for cytoplasm, the missed GO term is GO:0005524 (MF, ATP binding); and for mitochondrion, the missed GO term is GO:0005654 (CC, nucleoplasm). As can be seen from Section S1 of supplementary materials, the weights of these three GO terms for mLASSO in the aforementioned subcellular locations are negative and inconsiderable. Specifically, the weight of GO:0005829 for centrosome is -0.0045; the weight of GO:0005524 for cytoplasm is -0.0009 ; and the weight of GO:0005654 for mitochondrion is -0.0059 . Therefore, even though mLASSO selects them, these GO terms play insignificant roles in predictions. We notice that GO:0005829 (CC, cytosol) is the part of cytoplasm that does not contain organelles, which, in other words, has no direct correlation with centrosome. Because $\mathrm{mEN}$ tends to select correlated features together, it is reasonable that $\mathrm{mEN}$ does not select GO:0005829 for centrosome. Similar reasons are also applied to the other two cases. The remaining 11 subcellular locations have similar situations as Fig. 3(a), meaning that all of the essential GO terms selected by mLASSO are also selected by $\mathrm{mEN}$. The results suggest that mEN can select almost all the information selected by mLASSO, and more importantly, mEN can incorporate extra feature information missed by mLASSO.

\section{Significance of location-specific GO terms}

To quantitatively demonstrate how and to what extent essential GO terms contribute to the prediction of subcellular locations, we analyzed the location-specific weights $\left\{\tilde{\boldsymbol{\beta}}_{m}\right\}_{m=\{1, \ldots, M\}}$ defined in Eqs. 12 and 13 for the essential GO terms. ${ }^{8}$ As shown in Fig. 4, the analyses include (a) the number of non-zero weights for both algorithms, (b) the number of positive and negative weights for both algorithms, (c) distribution of weights for mLASSO and

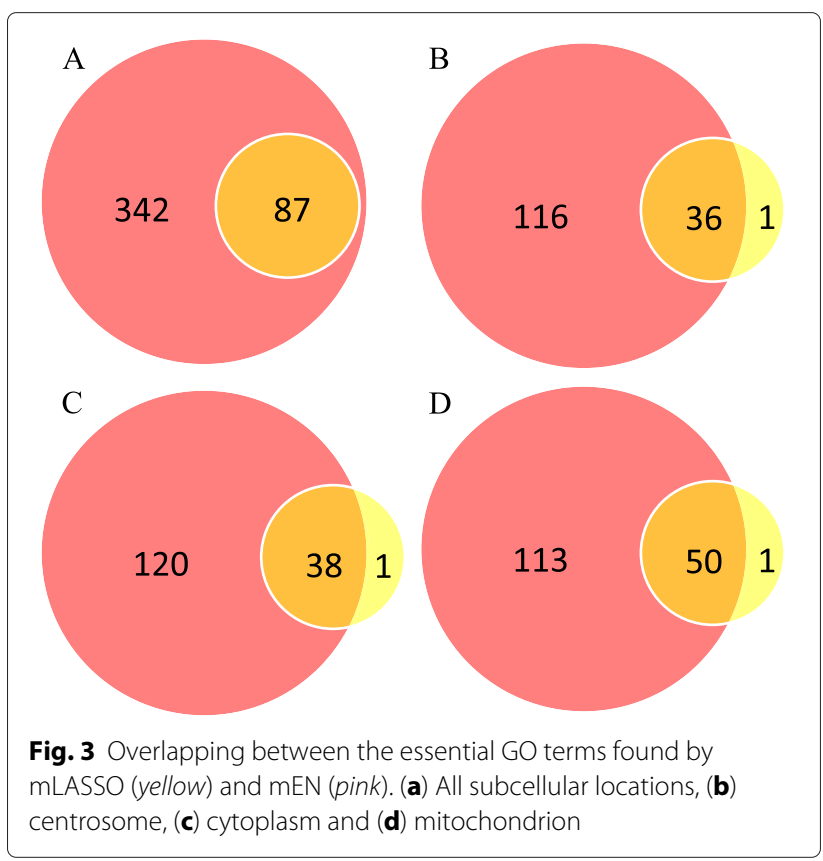




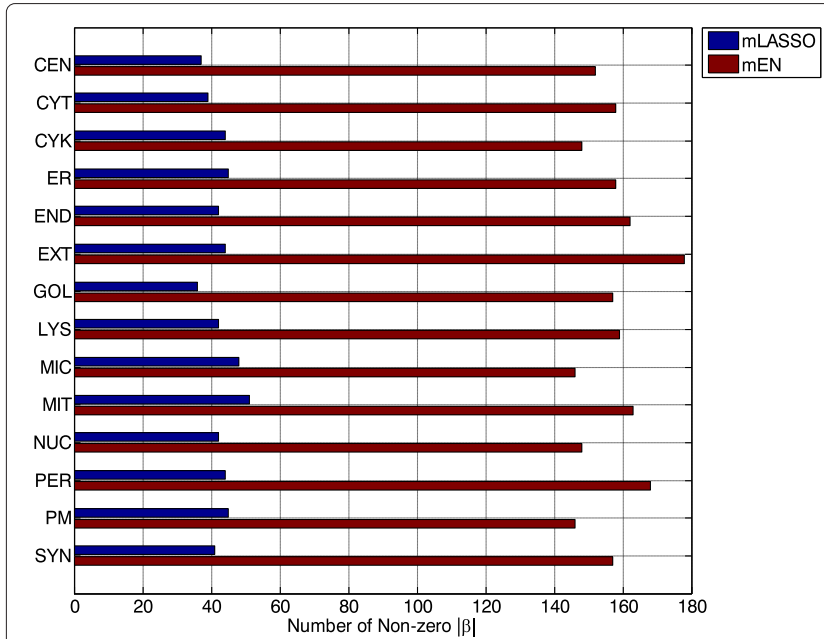

(a) Number of Non-zero Weights

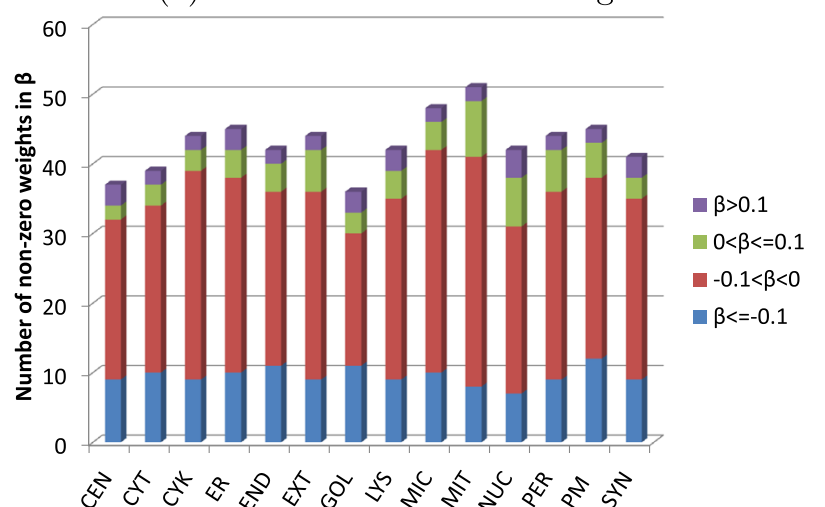

(c) Distribution of Weights in mLASSO

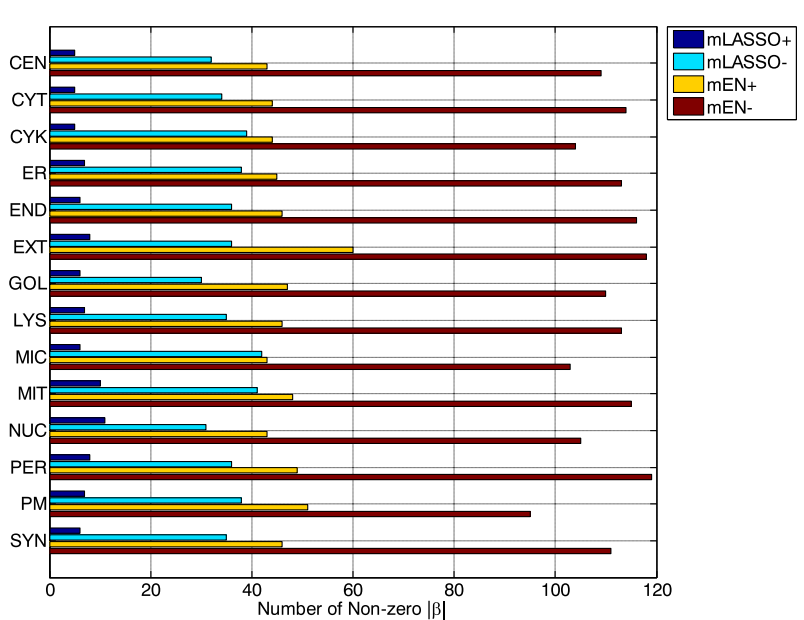

(b) Number of Positive and Negative Weights

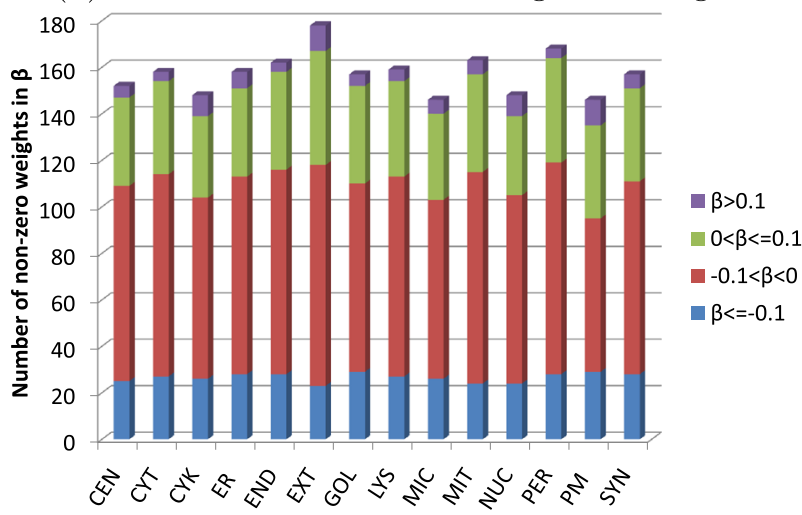

(d) Distribution of Weights in mEN

Fig. 4 Analysis of location-specific weights $\beta_{s, m}$ of the essential GO terms for mLASSO and mEN. (a) Number of non-zero weights for mLASSO and mEN; (b) number of positive and negative weights; (c) distribution of non-zero weights for mLASSO; (d) distribution of non-zero weights for mEN. CEN: centrosome; CYT: cytoplasm; CYK: cytoskeleton; ER: endoplasmic reticulum; END: endosome; EXT: extracellular; GOL: Golgi apparatus; LYS: lysosome; MIC: microsome; MIT: mitochondrion; NUC: nucleus; PER: peroxisome; PM: plasma membrane; SYN: synapse

(d) distribution of weights for mEN. For simplicity, $\beta_{s, m}$ is abbreviated as $\beta$ in the figures.

As can be seen from Fig. 4(a), for every subcellular location, the number of non-zero weights for $\mathrm{mEN}$ is larger than that for mLASSO, which is consistent with the results in the last section. In Fig. 4(b), we can see that for both mLASSO and $\mathrm{mEN}$, the number of positive weights in every subcellular location is much smaller than that of negative weights. This result suggests that the majority of essential GO terms are indicative of not residing in a particular subcellular location.

To further analyze the significance of the non-zero weights of essential GO terms, the weights are divided into four intervals:

$$
\left\{\begin{array}{l}
\beta \leq-0.1, \\
-0.1<\beta<0, \\
0<\beta \leq 0.1, \\
\beta>0.1 .
\end{array}\right.
$$

The distributions of the non-zero weights for mLASSO and mEN are shown in Fig. 4(c) and (d), respectively. From these figures, we can observe that the number of weights in the interval $\beta>0.1$ takes up the smallest percentage and most of the weights are within the interval of $-0.1<\beta<0$. This means that the number of essential GO terms indicating the presence of query proteins in a particular location is small. Nonetheless, when comparing Fig. 4(c) and (d), we found that the percentage of weights in the interval of $0<\beta \leq 0.1$ for $\mathrm{mEN}$ is larger than that for mLASSO, which suggests that there are more positive indicators for mEN than for mLASSO to indicate that a query protein locates in a particular subcellular location.

To further compare mEN and mLASSO, Fig. 5 shows the range of the non-zero weights for each subcellular location. Evidently, for almost all subcellular locations, the mean weights of $\mathrm{mEN}$ are larger than those of mLASSO, 


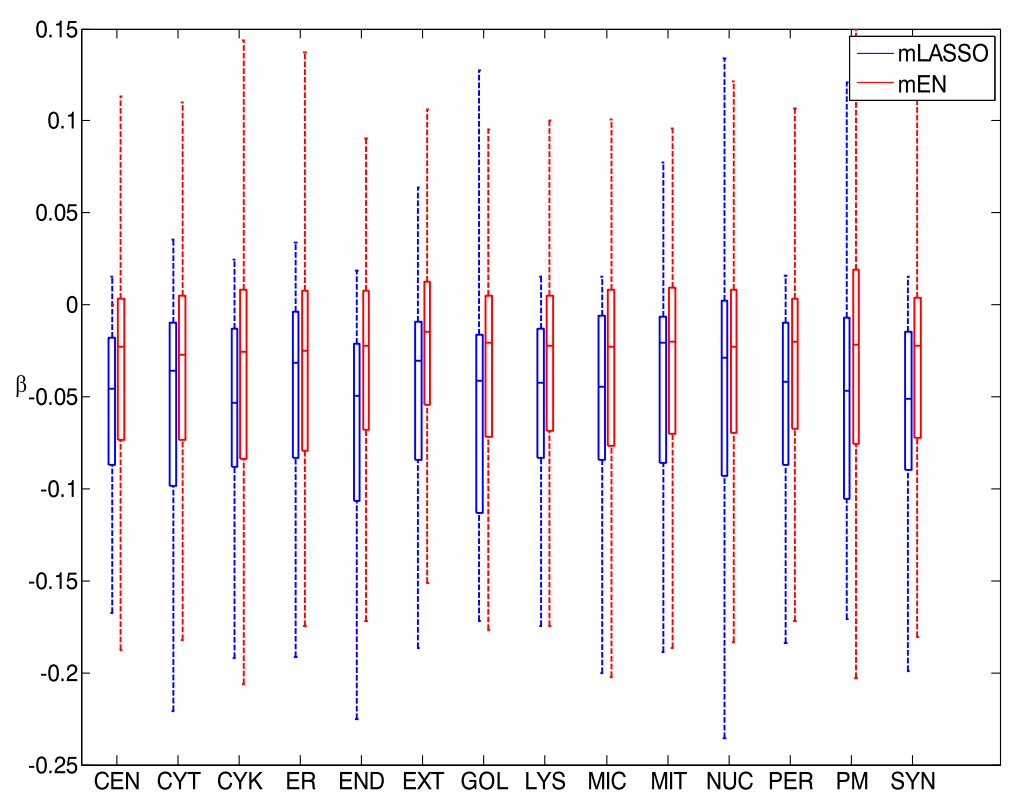

Fig. 5 The distribution of non-zero weights in each subcellular location for mLASSO and mEN. CEN: centrosome; CYT: cytoplasm; CYK: cytoskeleton; ER: endoplasmic reticulum; END: endosome; EXT: extracellular; GOL: Golgi apparatus; LYS: Iysosome; MIC: microsome; MIT: mitochondrion; NUC: nucleus; PER: peroxisome; PM: plasma membrane; SYN: synapse

which suggests that $\mathrm{mEN}$ are more capable of positively predicting a query protein than mLASSO.

We observe that among the essential GO terms, some have much larger absolute weights (i.e. $\left.\left|\beta_{s, m}\right|\right)$ than the rest, suggesting that they play more significant roles in making the predictions. Specifically, if the weight of an essential GO term for a particular subcellular location is larger than a certain positive threshold, it has higher confidence to indicate that the query protein resides in this subcellular location; on the contrary, if the weight is smaller than a certain negative threshold, it has higher confidence to indicate that the query protein does not belong to the corresponding subcellular location. We call these two kinds of GO terms as significantly essential GO terms.

\section{Circular networks for essential GO terms and subcellular locations}

To gain a comprehensive understanding of the relationships between the essential GO terms and the 14 subcellular locations for mLASSO and mEN, Fig. 6 shows the circular networks linking the essential GO terms and subcellular locations for four cases: (a) essential GO terms for mLASSO; (b) essential GO terms for mEN; (c) significantly essential GO terms for mLASSO; and (d) significantly essential GO terms for $\mathrm{mEN}$. In all figures, small green dots represent the GO terms and the large dots in different colors represent the 14 subcellular locations. A line connecting an essential GO term and a subcellular location means that the GO term contributes to the prediction of the subcellular location. On the other hand, if there is no connection between an essential GO term and a subcellular location, then this GO term does not provide any information about the presence or absence of a protein in this particular subcellular location. Starting from the top-left green dot to the bottom-left green dot in clockwise direction, the degree of overlapping among the lines gradually increases, meaning that the number of subcellular locations to which a GO term contributes also gradually increases. For example, in Fig. 6(a), the first 7 GO terms (GO:0007275, GO:0006915, GO:0006355, GO:0005643, GO:0005524, GO:0048471 and GO:0004674) are indicative of cytoplasm only, i.e., suggesting whether a protein belongs to cytoplasm or not. Similarly, GO:0005509 can only indicates whether a protein is located in endoplasmic reticulum or not. On the other hand, GO:0005815 is indicative for both centrosome and cytoskeleton; GO:0005635 contributes to the prediction of both cytoplasm and nucleus. More aggressively, the last several GO terms, such as GO:0016787, GO:0046872 and GO:0005515, contribute to the prediction of all of the 14 subcellular locations. These essential GO terms are indicators of whether a protein resides in one or more subcellular location(s) or not. Similar conclusions can be drawn from Fig. 6(b). Compared to Fig. 6(a, b) has more essential GO terms to indicate the presence or absence of a query protein in the corresponding subcellular location. For readers' convenience, all the 





essential GO terms found by mLASSO and mEN are listed in Section S3 and S4, respectively, of supplementary materials.

Figure 6(c) and (d) show the correlations between the significantly essential GO terms and the 14 subcellular locations (See the last section for the definition of significantly essential GO terms). There are 31 and 115 significantly essential GO terms shown in Fig. 6(c) and (d), respectively. As can be seen from Fig. 6(c), starting from the top-left to the bottom-left in clockwise direction, the first $12 \mathrm{GO}$ terms are indicative of single-location only. The remaining 19 significantly essential GO terms are multi-location indicators. For readers' convenience, all the essential GO terms found by mLASSO and mEN are listed in Sections S5 and S6, respectively, of supplementary materials.

Here we introduce the concept of the key GO terms, which are GO terms whose names are exactly the same as the names of subcellular locations according to the annotations. Interestingly, the key GO terms of some subcellular locations are both positive and negative indicators of multiple locations. For example, GO:0005813 is the key GO term for centrosome. A query protein with this term strongly indicates that it resides in centrosome, which is consistent with our results. However, $\mathrm{mEN}$ and mLASSO find that GO:0005813 is a negative indicator of cytoplasm, meaning that if a query protein is associated with this GO term, this protein is highly likely not to reside in cytoplasm. Actually, we have found (results not shown) that many significantly essential GO terms are not only positive indicators of some subcellular locations, but also negative indicators of other subcellular locations (indicating that proteins are unlikely to reside in particular subcellular locations). These GO terms allow us to find the proteins that cannot be colocated and the subcellular locations of these impossible combinations.

\section{Comparing with state-of-the-art predictors}

Table 1 compares the performance of mLASSO and mEN against several state-of-the-art multi-label predictors on the human benchmark dataset. To the best of our knowledge, iLoc-Hum [68] is the best state-ofthe-art predictor specializing for predicting multi-label human protein subcellular localization. ${ }^{9}$ mGOASVM [44] is not designed for predicting human protein subcellular localization, so we retrained it and applied the retrained mGOASVM to the human dataset. All of the predictors use some forms of GO vectors as features. From the classification perspective, iLoc-Hum use a multi-label KNN classifier; mGOASVM [44] uses a multi-label SVM classifier; and the proposed mLASSO and mEN use multi-label LASSO and EN classifiers, respectively.
As shown in Table 1, mEN outperforms mLASSO in terms of all performance metrics, and it performs better than iLoc-Hum and mGOASVM in terms of $O A A$, Accuracy, Precision, F1, Micro F1, Macro F1 and $H L$. Both mLASSO and mEN perform significantly better than iLoc-Hum. The OAA of mLASSO and mEN are $6 \%$ (absolute) and $4 \%$ higher than those of iLoc-Hum, respectively. When comparing with mGOASVM, the $O A A$ of $\mathrm{mEN}$ is around $2 \%$ (absolute) higher than that of mGOASVM, although a bit less than mGOASVM on the OLA and Recall. In terms of Accuracy, Precision, F1, Micro F1, Macro F1 and $H L$, mEN performs better than mGOASVM. The results suggest that the proposed $\mathrm{mEN}$ performs better than the state-of-the-art classifiers. The individual locative accuracies of mEN are remarkably higher than that of iLoc-Hum, and are comparable to mGOASVM. The superiority of mEN over mLASSO is possibly caused by the fact that $\mathrm{mEN}$ selects more relevant GO terms (features) than mLASSO, and that the features selected by the former almost contains all of the information selected by the latter, as demonstrated in Fig. 3 . This suggests that mLASSO is vulnerable to missing some important information when yielding parsimonious solutions.

\section{Predicting and interpreting novel proteins}

To further exemplify how mEN predicts and interprets the subcellular localization of proteins, we collected several novel proteins as test proteins. These proteins, which include both single- and multi-location proteins, were experimentally determined and were added to Swiss-Prot between 19-Feb-2014 and 07-Jan-2015. ${ }^{10}$ The novelty of these proteins can impartially demonstrate the prediction powers of our proposed predictors. Table 2 shows the prediction results of the 7 novel proteins by $\mathrm{mEN}$. As can be seen, although these proteins are totally new to our training dataset (created before 2009), all of them are correctly predicted, including one multi-location protein (E9PAV3). The essential GO terms that contribute to the prediction decisions are also shown in Table 2. A comparison between the essential GO terms in Fig. 6 and the last column in Table 2 reveals that not all of the essential GO terms contribute to the final predictions. For example, for the protein P0DMR3, only 8 out of 22 GO terms are useful for determining the subcellular localization. Interestingly, even if two proteins are predicted to the same subcellular location, the essential GO terms for the two proteins are not necessarily the same. For example, for D3DTV9 and C9JSJ3, although both of them are correctly predicted to locate in nucleus, their essential GO terms are completely different. And there is no significantly essential GO terms for the protein D3DTV9. This suggests that the predictions made by $\mathrm{mEN}$ do not always rely on significantly essential GO terms. 
Table 1 Comparing mLASSO and mEN with state-of-the-art multi-label predictors based on leave-one-out cross-validation on the human dataset

\begin{tabular}{|c|c|c|c|c|c|}
\hline \multirow{2}{*}{ Label } & \multirow{2}{*}{ Subcellular location } & \multicolumn{4}{|c|}{ LOOCV Locative Accuracy (LA) } \\
\hline & & iLoc-Hum [68] & mGOASVM [44] & mLASSO & $\mathrm{mEN}$ \\
\hline 1 & Centrosome & $56 / 77=0.727$ & $64 / 77=0.831$ & $42 / 77=0.546$ & $60 / 77=0.779$ \\
\hline 2 & Cytoplasm & $561 / 817=0.687$ & $683 / 817=0.836$ & $699 / 817=0.856$ & $683 / 817=0.836$ \\
\hline 3 & Cytoskeleton & $27 / 79=0.342$ & $44 / 79=0.557$ & $29 / 79=0.367$ & $32 / 79=0.405$ \\
\hline 4 & Endoplasmic reticulum & $166 / 229=0.725$ & $193 / 229=0.843$ & $194 / 229=0.847$ & $190 / 229=0.830$ \\
\hline 5 & Endosome & $1 / 24=0.042$ & $9 / 24=0.375$ & $1 / 24=0.042$ & $5 / 24=0.208$ \\
\hline 6 & Extracellular & $325 / 385=0.844$ & $344 / 385=0.894$ & $311 / 385=0.808$ & $314 / 385=0.816$ \\
\hline 7 & Golgi apparatus & $99 / 161=0.615$ & $131 / 161=0.814$ & $118 / 161=0.733$ & $128 / 161=0.795$ \\
\hline 8 & Lysosome & $56 / 77=0.727$ & $71 / 77=0.922$ & $62 / 77=0.805$ & $74 / 77=0.961$ \\
\hline 9 & Microsome & $7 / 24=0.292$ & $18 / 24=0.750$ & $1 / 24=0.042$ & $14 / 24=0.583$ \\
\hline 10 & Mitochondrion & $284 / 364=0.780$ & $339 / 364=0.931$ & $336 / 364=0.923$ & $336 / 364=0.923$ \\
\hline 11 & Nucleus & $918 / 1021=0.899$ & $931 / 1021=0.912$ & $922 / 1021=0.903$ & $923 / 1021=0.904$ \\
\hline 12 & Peroxisome & $20 / 47=0.426$ & $43 / 47=0.915$ & $34 / 47=0.723$ & $39 / 47=0.830$ \\
\hline 13 & Plasma membrane & $277 / 354=0.783$ & $288 / 354=0.814$ & $267 / 354=0.754$ & $266 / 354=0.751$ \\
\hline 14 & Synapse & $12 / 22=0.546$ & $12 / 22=0.546$ & $3 / 22=0.136$ & $13 / 22=0.591$ \\
\hline \multicolumn{2}{|c|}{ Overall Actual Accuracy $(O A A)$} & $2118 / 3106=0.682$ & $2251 / 3106=0.725$ & $2265 / 3106=0.729$ & $2307 / 3106=\mathbf{0 . 7 4 3}$ \\
\hline \multicolumn{2}{|c|}{ Overall Locative Accuracy (OLA) } & $2809 / 3681=0.763$ & $3170 / 3681=\mathbf{0 . 8 6 1}$ & $3019 / 3681=0.820$ & $3077 / 3681=0.836$ \\
\hline \multicolumn{2}{|c|}{ Accuracy } & - & 0.821 & 0.814 & 0.827 \\
\hline \multicolumn{2}{|c|}{ Precision } & - & 0.851 & 0.859 & 0.869 \\
\hline \multicolumn{2}{|c|}{ Recall } & - & 0.888 & 0.857 & 0.870 \\
\hline \multicolumn{2}{|l|}{ F1 } & - & 0.853 & 0.843 & 0.855 \\
\hline \multicolumn{2}{|c|}{ Micro F1 } & - & 0.835 & 0.826 & 0.837 \\
\hline \multicolumn{2}{|c|}{ Macro F1 } & - & 0.740 & 0.638 & 0.741 \\
\hline \multicolumn{2}{|l|}{$H L$} & - & 0.029 & 0.029 & 0.028 \\
\hline
\end{tabular}

"-" means the corresponding references do not provide the related metrics. Note that OAA is the most stringent and objective among all the metrics. Data in bold represent the best result of the corresponding measures among all predictors

Figure 7 demonstrates how researchers can use mEN to interpret the prediction results of query proteins. Figure 7 (a) shows the scores produced by Eq. 14 in descending order using the query protein D3DTV9 (Table 2) as input, where $(\mathrm{P})$ and $(\mathrm{F})$ stand for biological process and molecular function categories, respectively. Also, the columns "Weight" and "Term-Freq" represent non-zero elements of $\tilde{\boldsymbol{\beta}}_{m}^{\mathrm{en}}$ in Eq. 13 and $\boldsymbol{x}_{t}^{s}$ in Eq. 11, and the column "Feature Score" represents the product of Weight and Term-Freq. The higher the feature score, the more contribution is the corresponding GO term to the prediction result. Since all of the 14 scores are negative, the number of subcellular locations is predicted to be 1 and the subcellular location is determined by the maximum score, which corresponds to nucleus. The scores and weights for the essential GO terms in nucleus and endosome are also shown in the right panel of Fig. 7(a). ${ }^{11}$ As can be seen, only 5 out of 13 (See Table 2) essential GO terms contribute to the scores corresponding to nucleus. More interestingly, the top essential GO term (GO:0051607) belongs to biological process $(\mathrm{P})$, while the remaining 4 belong to molecular function (F) and none of them belongs to the cellular-component category. This suggests that $\mathrm{GO}$ terms from the categories of molecular function and biological process can also play key roles in determining the subcellular localization of proteins. Figure 7(b) shows the case for a multi-location protein (E9PAV3). Evidently, there are two positive scores, respectively determined by 6 and 4 essential GO terms. Thus, E9PAV3 is predicted to colocate in cytoplasm and nucleus. This demonstrates that $\mathrm{mEN}$ can predict multi-location proteins. Moreover, the sets of essential GO terms to determine the presence of E9PAV3 in cytoplasm and nucleus are different.

\section{Discussion}

\section{Mapping non-essential GO terms to essential GO terms}

Some researchers are concerned that not all proteins (especially those novel proteins) are associated with essential GO terms selected by mLASSO (or mEN). In this case, the feature vectors for these proteins will be null, which 
Table 2 Prediction results of 7 novel proteins by mEN

\begin{tabular}{|c|c|c|c|c|c|}
\hline$A C$ & Date of creation & Ground-truth location(s) & Prediction results & $\begin{array}{l}\text { GO total } \\
\text { number }\end{array}$ & Essential GO terms \\
\hline D3DTV9 & 26-Nov-2014 & Nucleus & Nucleus & 13 & $\begin{array}{l}\text { GO:0000166, GO:0016787, } \\
\text { GO:0003676, GO:0003723, } \\
\text { GO:0004386, GO:0046872, } \\
\text { GO:0051607, GO:0005524 }\end{array}$ \\
\hline E9PAV3 & 19-Feb-2014 & Cytoplasm, Nucleus & Cytoplasm, Nucleus & 9 & $\begin{array}{l}\text { GO:0015031, GO:0003677, } \\
\text { GO:0005634, GO:0005737, } \\
\text { GO:0006351, GO:000635, } \\
\text { GO:0006810 }\end{array}$ \\
\hline B7ZW38 & 26-Nov-2014 & Nucleus & Nucleus & 5 & $\begin{array}{l}\text { GO:0000166, GO:0030529, } \\
\text { GO:0003676, GO:0003723, } \\
\text { GO:0005634 }\end{array}$ \\
\hline PODMR3 & 07-Jan-2015 & Cytoplasm & Cytoplasm & 22 & $\begin{array}{l}\text { GO:0000166, GO:0016740, } \\
\text { GO:0016874, GO:0003824, } \\
\text { GO:0046872, GO:0005524, } \\
\text { GO:0005575, GO:0008152 }\end{array}$ \\
\hline PODML3 & 09-Jul-2014 & Extracellular & Extracellular & 6 & $\begin{array}{l}\text { GO:0046872, GO:0005179, } \\
\text { GO:0005576, GO:0007165 }\end{array}$ \\
\hline PODMNO & 03-Sep-2014 & Cytoplasm & Cytoplasm & 16 & $\begin{array}{l}\text { GO:0016740, GO:0030968, } \\
\text { GO:0044267, GO:0044281, } \\
\text { GO:0005737, GO:0005829, } \\
\text { GO:0006629, GO:0006805 }\end{array}$ \\
\hline C9JSJ3 & 29-Oct-2014 & Nucleus & Nucleus & 4 & $\begin{array}{l}\text { GO:0003677, GO:0005634, } \\
\text { GO:0006351, GO:0006355 }\end{array}$ \\
\hline
\end{tabular}

AC: UniProtKB accession number; Ground-truth location(s): the experimentally-validated actual subcellular location(s); GO Total Number: the total number of GO terms retrieved for a given query protein

\begin{tabular}{|c|c|c|c|c|c|}
\hline SCL & Score & & & & \\
\hline NUC & -0.0527 & $\begin{array}{c}\text { Essential GO } \\
\text { Term }\end{array}$ & $\begin{array}{l}\text { Feature } \\
\text { score }\end{array}$ & weight & $\begin{array}{l}\text { Term- } \\
\text { Freq }\end{array}$ \\
\hline CYT & -0.2111 & GO:0051607 (P) & 0.0892 & 0.0892 & 1 \\
\hline PM & -0.3005 & GO:0003723 (F) & 0.0714 & 0.0357 & 2 \\
\hline GOL & -0.3360 & GO:0000166 (F) & -0.0528 & -0.0528 & 1 \\
\hline EXT & -0.3533 & GO:0016787 (F) & -0.0892 & -0.0892 & $\begin{array}{l}1 \\
1\end{array}$ \\
\hline ER & -0.3594 & \multirow{4}{*}{\multicolumn{4}{|c|}{$\cdot$}} \\
\hline MIT & -0.3937 & & & & \\
\hline LYS & -0.4030 & & & & \\
\hline MIC & -0.4249 & & & & \\
\hline CYK & -0.4276 & $\begin{array}{l}\text { Essential GO } \\
\text { Term }\end{array}$ & $\begin{array}{l}\text { Feature } \\
\text { score }\end{array}$ & weight & $\begin{array}{l}\text { Term- } \\
\text { Freq }\end{array}$ \\
\hline SYN & -0.4276 & GO:0003676 (F) & -0.0322 & -0.0322 & 1 \\
\hline CEN & -0.4280 & GO:0000166 (F) & -0.0714 & -0.0714 & 1 \\
\hline PER & -0.4328 & GO:0003723 (F) & -0.0866 & -0.0433 & 2 \\
\hline END & $-0.4594^{\prime}$ & $\begin{array}{l}\mathrm{G}: 00408 / 2(\mathrm{~F}) \\
\mathrm{GO}: 0016787 \text { (F) }\end{array}$ & $\begin{array}{l}-0.1016 \\
-0.1676\end{array}$ & $\begin{array}{l}-0.1016 \\
-0.1676\end{array}$ & $\begin{array}{l}1 \\
1\end{array}$ \\
\hline
\end{tabular}

(a) Predicting Protein D3DTV9

\begin{tabular}{|c|c|c|c|c|c|}
\hline SCL & Score & & & & \\
\hline CYT & 0.3997 V & $\begin{array}{l}\text { Essential Go } \\
\text { Term }\end{array}$ & $\begin{array}{l}\text { Feature } \\
\text { score }\end{array}$ & weight & $\begin{array}{l}\text { Term- } \\
\text { Freq }\end{array}$ \\
\hline NUC & 0.2675 & GO:0005737 (C) & 1.0749 & 0.3583 & 3 \\
\hline ER & -1.0203 & GO:0003677 (F) & -0.0072 & -0.0072 & 1 \\
\hline GOL & -10576 & GO:0006355 (P) & -0.0168 & -0.0168 & 1 \\
\hline GOL & $-1.05 / 0$ & GO:0006810 (P) & -0.0218 & -0.0218 & 1 \\
\hline END & -1.0793 & GO:0006351 (P) & -0.1134 & -0.1134 & 1 \\
\hline MIT & -1.0852 & GO:0005634 (C) & -0.5160 & -0.1720 & 3 \\
\hline CYK & -1.1165 & Essential GO & Feature & weight & Term- \\
\hline SYN & -1.1195 & Term & score & & Freq \\
\hline PER & -1.1249 & $\begin{array}{l}\text { GO:0005634 (C) } \\
\text { GO:0006810 (P) }\end{array}$ & 0.8844 & 0.2948 & 3 \\
\hline LYS & -1.1261 & GO:0015031 (P) & -0.0957 & $\begin{array}{l}-0.0415 \\
-0.0957\end{array}$ & 1 \\
\hline MIC & -1.1366 & GO:0005737 (C) & -0.4797 & -0.1599 & 3 \\
\hline EXT & -1.1425 & & . & & \\
\hline CEN & -1.1459 & & : & & \\
\hline PM & -1.1566 & & . & & \\
\hline
\end{tabular}

(b) Predicting Protein E9PAV3

Fig. 7 Examples showing how mEN interprets subcellular localization of (a) a single-location protein (D3DTV9) and (b) a multi-location protein (E9PAV3). SCL: subcellular location; Score: the score determined in Eq. 14; Feature Score: the score that each essential GO term contributes to the final prediction; Term-freq: the frequency of occurrence of an essential GO term; C: cellular component; $F$ : molecular function; $P$ : biological process; CEN: centrosome; CYT: cytoplasm; CYK: cytoskeleton; ER: endoplasmic reticulum; END: endosome; EXT: extracellular; GOL: Golgi apparatus; LYS: Iysosome; MIC: microsome; MIT: mitochondrion; NUC: nucleus; PER: peroxisome; PM: plasma membrane; SYN: synapse 
is detrimental for prediction performance. To address this problem, we have proposed a hierarchical-information based approach to map the non-essential GO terms to essential GO terms. Because the GO terms in each taxonomy (cellular components, molecular functions or biological processes) are hierarchically organized within a directed acyclic graph (DAG), GO terms in the same taxonomy are not independent with each other. In this case, non-essential GO terms are hierarchically correlated with essential GO terms within the same taxonomy. Therefore, it is feasible and also conducive to map the non-essential GO terms to the essential GO terms based on their hierarchical structural relationships.

We adopt our recently proposed GO mapping method to perform the mapping. Here, we briefly outline the procedure. For implementation details, readers may refer to Eqs. 5 and 6 of [69]. Denote $G$ as a GO term (either non-essential or essential) and $E$ as an essential GO term. Assume that $G$ is one of the GO terms associated with a protein and that the number of occurrences of $G$ for that protein is $f_{G}$. Then, mapping $G$ to $E$ is equivalent to finding the contribution of $G$ to the effective number of occurrences of $E$, where the contribution is computed from the depth distance between $G$ and $E$ as shown in Algorithm 1 . The effective number of occurrences of $E$ is the sum of the contribution of all GO terms associated with the query protein. This procedure is repeated for every essential GO terms to form a vector comprising the effective numbers of occurrences of all essential GO terms. This GO mapping method effectively solves the null-vector problem, because as long as one of the GO term associated with the query protein is close enough to any essential GO term in the GO DAG, the resulting vector will not be null.

Table 3 investigates the impacts of the hierarchicalinformation based (HIB) technique on mLASSO and $\mathrm{mEN}$ based on LOOCV on the human dataset. As can be seen, the HIB technique can improve the performance of mLASSO in terms of all performance metrics. On the contrary, mEN with HIB performs slightly worse than mEN without HIB. This is understandable because compared to mEN, mLASSO selects fewer GO terms so that information in some of the discarded GO terms is lost. The HIB method can partially retain this lost information in the HIB vectors (Eq. 5 of [69]) through the structural relationship between the discarded terms and the selected terms, leading to improved performance. On the other hand, because mEN selects more essential GO terms and thus less information is lost, the HIB technique is less helpful to mEN. The performance of $\mathrm{mEN}$ with the HIB technique is even slightly worse than that of $\mathrm{mEN}$ without HIB due to different representations of the feature information. More interestingly, except for Macro F1, the performance of mLASSO with HIB outweighs that of $\mathrm{mEN}$ in all performance metrics.

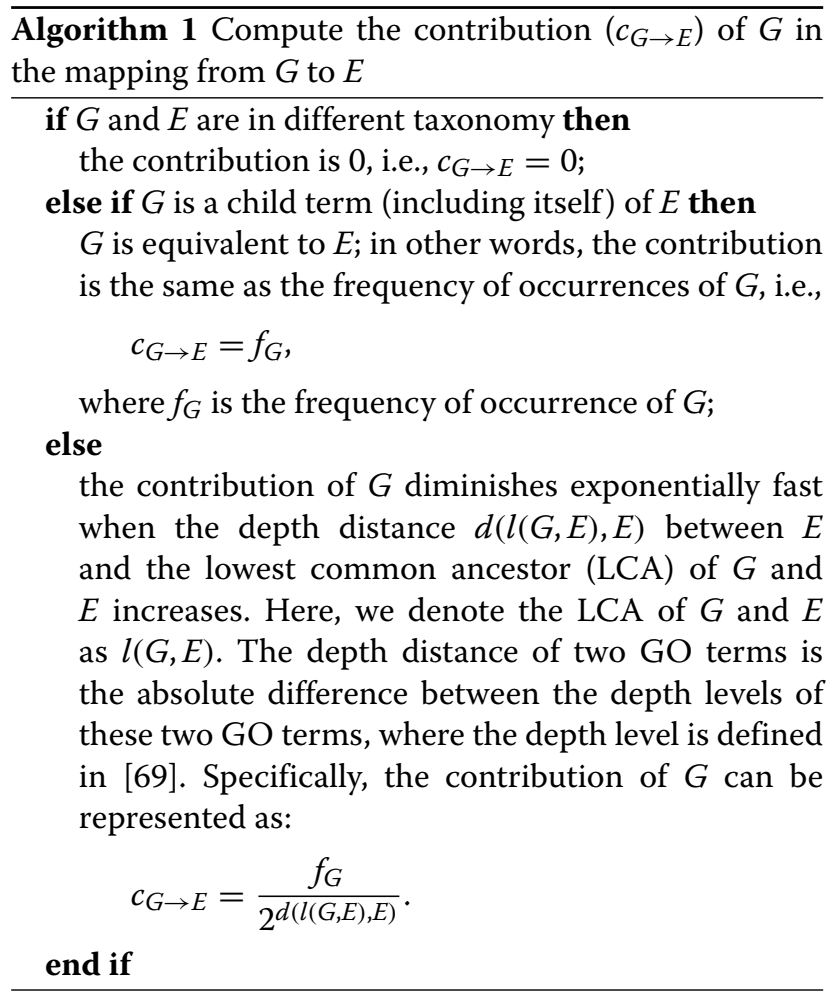

\section{Categorical significance of $\mathrm{GO}$ terms on prediction}

To investigate the contributions of GO terms from different categories to the prediction performance of mLASSO and $\mathrm{mEN}$, we have compared the performance of using GO terms for the following cases: (1) All: all GO terms are used; (2) $C C+M F$ : GO terms from cellular components (CC) and molecular functions (MF) are used; (3) $C C+B P$ : GO terms from CC and biological processes (BP); and (4) $M F+B P: \mathrm{GO}$ terms from $\mathrm{MF}$ and $\mathrm{BP}$ are used. The results are shown in Table 4 . As can be seen, for both mLASSO

Table 3 Impacts of the hierarchical-information based (HIB) technique on $\mathrm{mLASSO}$ and $\mathrm{mEN}$ based on leave-one-out cross-validation (LOOCV) on the human dataset

\begin{tabular}{lllllll}
\hline \multirow{2}{*}{ Measures } & \multicolumn{3}{c}{$\mathrm{mLASSO}$} & & \multicolumn{2}{c}{$\mathrm{mEN}$} \\
\cline { 2 - 3 } \cline { 6 - 6 } \cline { 5 - 6 } OAA & without HIB & with HIB & & without HIB & with HIB \\
OLA & 0.729 & $\mathbf{0 . 7 4 8}$ & & 0.743 & 0.742 \\
Accuracy & 0.820 & $\mathbf{0 . 8 4 6}$ & & 0.836 & 0.825 \\
Precision & 0.859 & $\mathbf{0 . 8 3 3}$ & & 0.827 & 0.821 \\
Recall & 0.857 & $\mathbf{0 . 8 7 4}$ & & 0.869 & 0.866 \\
F1 & 0.843 & $\mathbf{0 . 8 7 9}$ & & 0.870 & 0.860 \\
Micro F1 & 0.826 & $\mathbf{0 . 8 6 2}$ & & 0.855 & 0.849 \\
Macro F1 & 0.638 & $\mathbf{0 . 8 4 4}$ & & 0.837 & 0.831 \\
HL & 0.029 & 0.676 & & $\mathbf{0 . 7 4 1}$ & 0.667 \\
\hline
\end{tabular}

Note that $O A A$ is the most stringent and objective among all the metrics. Data in bold represent the best result of the corresponding measures among all predictors 
Table 4 Significance of GO terms from different categories on the performance of mLASSO and mEN based on leave-one-out cross-validation (LOOCV) on the human dataset

\begin{tabular}{|c|c|c|c|c|c|c|c|c|}
\hline \multirow{2}{*}{ Measures } & \multicolumn{4}{|c|}{$\mathrm{mLASSO}$} & \multicolumn{4}{|c|}{$\mathrm{mEN}$} \\
\hline & All & $C C+M F$ & $C C+B P$ & $\mathrm{MF}+\mathrm{BP}$ & All & $C C+M F$ & $C C+B P$ & $\mathrm{MF}+\mathrm{BP}$ \\
\hline$O A A$ & 0.729 & 0.662 & 0.654 & 0.385 & 0.743 & 0.621 & 0.640 & 0.440 \\
\hline OLA & 0.820 & 0.726 & 0.715 & 0.436 & 0.836 & 0.686 & 0.701 & 0.492 \\
\hline Accuracy & 0.814 & 0.733 & 0.724 & 0.446 & 0.827 & 0.690 & 0.709 & 0.506 \\
\hline Precision & 0.859 & 0.782 & 0.773 & 0.500 & 0.869 & 0.739 & 0.760 & 0.560 \\
\hline Recall & 0.857 & 0.759 & 0.747 & 0.457 & 0.870 & 0.712 & 0.730 & 0.521 \\
\hline$F 1$ & 0.843 & 0.758 & 0.748 & 0.469 & 0.855 & 0.713 & 0.733 & 0.528 \\
\hline Micro F1 & 0.826 & 0.750 & 0.741 & 0.462 & 0.837 & 0.711 & 0.728 & 0.516 \\
\hline Macro F1 & 0.638 & 0.435 & 0.426 & 0.212 & 0.741 & 0.410 & 0.427 & 0.346 \\
\hline$H L$ & 0.029 & 0.041 & 0.042 & 0.086 & 0.028 & 0.047 & 0.044 & 0.078 \\
\hline
\end{tabular}

Note that $O A A$ is the most stringent and objective among all the metrics. CC: cellular components; MF: molecular functions; BP: biological processes. Data in bold represent the best result of the corresponding measures among all predictors

and mEN, Case (1) performs the best among all the four cases, due to using GO terms from all of the three categories. The results indicate that GO terms from all of the three categories are conducive to the predictions. Moreover, the performance of Cases (2) and (3) remarkably surpass that of Case (4), which suggests that CC GO terms are more far more significant than GO terms from the other two categories for the predictions. Besides, although Case (4) performs the worst among the four cases, it also demonstrates that GO terms from MF and BP are also useful for the predictions.

\section{Conclusions}

In this paper, we proposed and compared two sparse multi-label predictors, namely mLASSO and mEN, which can predict as well as interpret the subcellular localization(s) of both single- and multi-location proteins. Given a query protein, its feature vector is constructed by exploiting the GO frequency information in the ProSeqGO database. By using the one-vs-rest LASSO and EN classifiers, 87 and 429 out of $8,000+$ GO terms are selected, respectively. Based on these selected essential GO terms, the interpretability is analyzed for both algorithms.

This paper has the following key contributions: (1) Both mEN and mLASSO are interpretable and perform remarkably better than existing state-of-the-art predictors; (2) mEN selects more relevant GO terms than mLASSO, and meanwhile outperforms mLASSO; (3) Experimental results for both methods are consistent with biological annotations, i.e., the key GO terms play greater roles in determining subcellular localization of proteins; (4) Like cellular-component GO terms, GO terms from the categories of molecular functions and biological processes also contribute to the prediction; (5) Essential GO terms can be either single-location contributive or multi-location contributive to the prediction, and the contributions can be positive on a subcellular location while be negative on other subcellular locations. For readers' convenience, the SpaPredictor web-server and the supplementary materials of this paper are available online at http://bioinfo.eie.polyu.edu.hk/SpaPredictorServer/.

\section{Methods \\ Feature extraction \\ 1) Creation of compact databases}

The applicability of existing GO-based approaches is limited by the availability of GO information for query proteins, especially for novel proteins. Conventionally, given a query protein, if its accession number (AC) cannot be associated with any GO term in the GOA database, BLAST [53] was used to retrieve its top homologous protein which is supposed to be annotated in the GOA database, and thus whose AC can be associated with a set of GO terms. In this case, the homologous GO information can be transferred to the query protein. However, this strategy will become ineffective when no GO information can be retrieved from the top homolog. In such case, some predictors use back-up methods that rely on other features, such as pseudo-amino-acid composition [14] and sorting signals [70]; some predictors [34, 44] use a successive-search strategy to avoid null GO vectors. Nonetheless, these strategies may lead to poor performance and increase computation and storage complexity.

To address this problem, similar to our earlier work $[46,71]$, we created two small yet efficient databases: ProSeq and ProSeq-GO. The former is a sequence database extracted from the Swiss-Prot database and the latter is a GO-term database extracted from the GOA database. Detailed descriptions of the procedures can be found in [46]. By using ProSeq and ProSeq-GO, we can not only guarantee that every query protein can associate 
with at least one GO term, but also significantly reduce the memory consumption.

\section{2) Construction of GO vectors}

The construction of feature vectors involves two steps: (1) retrieval of GO terms; and (2) construction of GO vectors.

For the retrieval of GO terms, given a query protein, its amino acid sequence is presented to BLAST [53] to find its homologs in the ProSeq database. The homologous ACs are then used as keys to search against the ProSeq-GO database.

For the construction of $\mathrm{GO}$ vectors, given a dataset, the $\mathrm{GO}$ terms of all of its proteins are retrieved by the procedures described above. Because term-frequency (TF) based GO vectors [34, 44] are found to perform better than the conventional 1-0 vectors, we adopted the TF method to construct $G O$ vectors. Specifically, suppose the number of distinct $\mathrm{GO}$ terms for the dataset of interest is $T$, then the GO vector $\mathbf{q}_{i}$ of the $i$-th protein $\mathbb{Q}_{i}$ is defined as:

$$
\mathbf{q}_{i}=\left[f_{i, 1}, \ldots, f_{i, j}, \ldots, f_{i, T}\right]^{\top},
$$

where $f_{i, j}$ is the number of occurrences of the $j$-th GO term (term-frequency) in the $i$-th protein sequence. Detailed information about $\mathrm{GO}$ vectors can be found in $[34,44]$.

\section{Multi-label sparse-regression based classifiers}

An interesting and useful property of sparse regression models is that they can produce "parsimonious" solutions that enable us to find a set of features that are the most relevant to the problem (target variables) being addressed. Usually, sparse regressions are achieved by imposing regularized constraints on the features. Two common linear sparse regression models are LASSO [72] and elastic net (EN) [67]. The former is short for Least Absolute Shrinkage and Selection Operator, which is an $L_{1}$-regularized linear regression model. The $L_{1}$ constraint forces the weights of some features to exactly zero [73], and hence LASSO can automatically select relevant features. The latter is an $\left(L_{1}+L_{2}\right)$-regularized linear regression model. The convex combination of $L_{1}$ and $L_{2}$ penalties can yield sparse representations similar to LASSO, while encouraging correlated features to be selected or deselected together [67]. LASSO can be regarded as a special case of EN, which is explained in the following section.

LASSO has been applied to many bioinformatics domains, such as gene regulation network analysis [73], microRNA-target regulatory network construction [74], inflammation-cancer relationship analysis [75] and plant gene detection [76]. EN has been extensively used in various aspects of computational biology, such as genetic trait prediction [77], ICU mortality risk detection [78], single nucleotide polymorphism (SNP) selection [79], etc.

\section{1) Objective functions of sparse regressions}

Sparse regressions are applicable to classification. Suppose for a two-class single-label problem, we are given a set of training data $\left\{\mathbf{x}_{i}, y_{i}\right\}_{i=1}^{N}$, where $\mathbf{x}_{i} \in \mathcal{R}^{T}$ and $y_{i} \in$ $\{-1,1\}$. In our case, $\mathbf{x}_{i}=\mathbf{q}_{i}$, where $\mathbf{q}_{i}$ is defined in Eq. 5 .

Generally speaking, a LASSO model is to impose an $L_{1}$ regularization to ordinary least squares (OLS):

$l^{\mathrm{lasso}}(\boldsymbol{\beta})=\sum_{i=1}^{N}\left(y_{i}-f\left(\mathbf{x}_{i}\right)\right)^{2}=\sum_{i=1}^{N}\left(y_{i}-\varepsilon_{0}-\sum_{j=1}^{T} \beta_{j} x_{i, j}\right)^{2}$,

subject to

$$
\sum_{j=1}^{T}\left|\beta_{j}\right| \leq t
$$

where $\boldsymbol{\beta}=\left[\beta_{1}, \ldots, \beta_{j}, \ldots, \beta_{T}\right]^{\top}$ is the LASSO vector to be optimized, $t>0$ is a parameter controlling the shrinkage level applied to $\boldsymbol{\beta}, \varepsilon_{0}$ is a bias, ${ }^{12}$ and $x_{i, j}$ is the $j$-th element of $\mathbf{x}_{i}$. Equation 6 is equivalent to minimizing the following equation:

$$
l^{\text {lasso }}(\boldsymbol{\beta})=\sum_{i=1}^{N}\left(y_{i}-\boldsymbol{\beta}^{\top} \mathbf{x}_{i}\right)^{2}+\lambda \sum_{j=1}^{T}\left|\beta_{j}\right|,
$$

where $\lambda>0$ is a penalized parameter controlling the degree of regularization. Equation 7 is a convex optimization problem, and can be efficiently solved. We adopted the least angle regression (LARS) method to solve this problem. Detailed descriptions of the LARS algorithm can be found in [80].

EN is to impose an $\left(L_{1}+L_{2}\right)$-style regularization on Eq. 6. Thus, the object function of EN is:

$$
l^{\mathrm{en}}(\boldsymbol{\beta})=\sum_{i=1}^{N}\left(y_{i}-\boldsymbol{\beta}^{\top} \mathbf{x}_{i}\right)^{2}+\lambda \sum_{j=1}^{T}\left|\beta_{j}\right|+\gamma \sum_{j=1}^{T} \beta_{j}{ }^{2},
$$

where $\lambda>0$ and $\gamma>0$ are the penalty parameters controlling the ridge regression penalty and lasso penalty, respectively. As can be seen, when $\lambda=0$, Eq. 8 becomes simple ridge regression; when $\gamma=0$, Eq. 8 is exactly the same as Eq. 7. Besides, by simple transformation, Eq. 8 can be converted to an equivalent LASSO-style problem on augmented data [67]. Because of this property, Eq. 8 can be solved by the same way as LASSO by absorbing the $L_{2}$-norm term into the objective function. Detailed descriptions of the solutions can be found in [67]. In this work, the LASSO and the elastic net algorithms were implemented by using the functions lasso.m and elasticnet.m, respectively, in the SpaSM package [81]. This package can be downloaded from http://www2.imm.dtu. $\mathrm{dk} /$ projects/spasm/. 


\section{2) Multi-label LASSO/EN for feature selection}

In an $M$-class multi-label problem, the training data set is written as $\left\{\mathbf{x}_{i}, \mathcal{Y}_{i}\right\}_{i=1}^{N}$, where $\mathbf{x}_{i} \in \mathcal{R}^{T}$ and $\mathcal{Y}_{i} \subset$ $\{1,2, \ldots, M\}$ is a set which may contain one or more labels.

For the multi-label LASSO (EN), $M$ independent binary one-vs-rest LASSOs (ENs) are trained, one for each class. The labels $\left\{\mathcal{Y}_{i}\right\}_{i=1}^{N}$ are converted to transformed labels [44] $y_{i, m} \in\{-1,1\}$, where $i=1, \ldots, N$, and $m=1, \ldots, M$. Then, the LASSO and EN estimate vectors for the $m$-th class is given by:

$$
\hat{\boldsymbol{\beta}}_{m}^{\text {lasso }}=\underset{\boldsymbol{\beta}_{m}}{\arg \min }\left\{\sum_{i=1}^{N}\left(y_{i, m}-\boldsymbol{\beta}_{m}^{\boldsymbol{\top}} \mathbf{x}_{i}\right)^{2}+\lambda_{m} \sum_{j=1}^{T}\left|\beta_{j, m}\right|\right\},
$$

and

$$
\hat{\boldsymbol{\beta}}_{m}^{\mathrm{en}}=\underset{\boldsymbol{\beta}_{m}}{\arg \min }\left\{\sum_{i=1}^{N}\left(y_{i, m}-\boldsymbol{\beta}_{m}^{\top} \mathbf{x}_{i}\right)^{2}+\lambda_{m} \sum_{j=1}^{T}\left|\beta_{j, m}\right|+\gamma_{m} \sum_{j=1}^{T} \beta_{j, m}{ }^{2}\right\},
$$

respectively. In Eq. $9, m=1, \ldots, M,\left\{y_{i, m}\right\}_{i=1}^{N} \in\{-1,1\}$, and $\lambda_{m}$ is the penalized parameter for the $m$-th class. And in Eq. $10, m=1, \ldots, M,\left\{y_{i, m}\right\}_{i=1}^{N} \in\{-1,1\}, \lambda_{m}$ and $\gamma_{m}$ are the $L_{1}$ penalized parameter and the $L_{2}$ penalized parameter for the $m$-th class, respectively. Since $L_{1}$ regularization tends to force some weights $\left\{\beta_{j, m}\right\}_{j=1}^{T}$ for the $m$-th class to exactly zero, both LASSO and EN can be used for feature selection. The difference between LASSO and EN is the degree of parsimoniousness in the solution.

The GO vectors obtained from Eq. 5 are used for training multi-label one-vs-rest LASSO classifiers. For an $M$-class problem (here $M$ is the number of subcellular locations), $M$ independent binary LASSO classifiers are trained, one for each class. After training, the union of those GO terms whose weights are not zero in any one of the $M$ classes constitutes the selected features. LASSO can impressively remove those irrelevant features (or GO terms). Suppose $S$ out of the $T$ weights are nonzero. They are defined as $\left\{\beta_{s, m}\right\}_{s=\{1, \ldots, S\}, m=\{1, \ldots, M\}}$ and their corresponding GO terms are called essential GO terms. In fact, in our experiments, through the proposed multi-label LASSO classifiers, 87 out of $8110 \mathrm{GO}$ terms were selected. This means that only around $1 \%$ of the GO terms are essential GO terms and the weights for about $99 \%$ of the 8110 GO terms are exactly zero.

Similar procedures were applied to multi-label EN. Through the proposed multi-label EN classifiers, 429 out of $8110 \mathrm{GO}$ terms were selected. This means that around $5 \%$ of the GO terms are essential GO terms and the weights for about $95 \%$ of the $8110 \mathrm{GO}$ terms are exactly zero.

\section{3) Multi-label LASSO/EN for classification}

Besides feature selection, LASSO and EN can also be used for classification. Specifically, given the $t$-th query protein $\mathbb{Q}_{t}$, the feature vector $\mathbf{x}_{t} \in \mathcal{R}^{T}$ defined in Eq. 5 is obtained. Then, the elements of $\mathbf{x}_{t}$ with non-zero weights $\beta_{j, m}$ (in Eq. 9 for LASSO and in Eq. 10 for EN) are selected to form a low-dimensional feature vector represented by $\mathbf{x}_{t}^{s} \in \mathcal{R}^{S}$, where $S<T$ is the number of essential GO terms. Similarly, for an $M$-class problem, $M$ independent binary LASSO (EN) classifiers are trained, one for each class. Then, the score of the $m$-th LASSO (EN) is:

$$
s_{m}\left(\mathbb{Q}_{t}\right)=\tilde{\boldsymbol{\beta}}_{m}^{\top} \mathbf{x}_{t}^{s},
$$

where $\tilde{\boldsymbol{\beta}}_{m}$ for LASSO and EN are given by

$$
\tilde{\boldsymbol{\beta}}_{m}^{\text {lasso }}=\underset{\boldsymbol{\alpha}_{m}}{\arg \min }\left\{\sum_{i=1}^{N}\left(y_{i, m}-\boldsymbol{\alpha}_{m}^{\top} \mathbf{x}_{i}^{s}\right)^{2}+\lambda_{m} \sum_{j=1}^{S}\left|\alpha_{j, m}\right|\right\},
$$

and

$$
\tilde{\boldsymbol{\beta}}_{m}^{\text {en }}=\underset{\boldsymbol{\alpha}_{m}}{\arg \min }\left\{\sum_{i=1}^{N}\left(y_{i, m}-\boldsymbol{\alpha}_{m}^{\top} \mathbf{x}_{i}^{s}\right)^{2}+\lambda_{m} \sum_{j=1}^{S}\left|\alpha_{j, m}\right|+\gamma_{m} \sum_{j=1}^{S} \alpha_{j, m}{ }^{2}\right\},
$$

respectively, where $\boldsymbol{\alpha}_{m}=\left[\alpha_{1, m}, \ldots, \alpha_{j, m}, \ldots, \alpha_{S, m}\right]^{\top}$ is the weight vector to be optimized and $\mathbf{x}_{i}^{s} \in \mathcal{R}^{S}$ is the feature vector for the $i$-th training protein. Note that $\tilde{\boldsymbol{\beta}}_{m}$ in both equations are obtained based only on the training data.

To predict the subcellular locations of datasets containing both single-label and multi-label proteins, a decision scheme for multi-label LASSO (EN) classifiers should be used. Unlike the single-label problem where each protein has one predicted label only, a multi-label protein should have more than one predicted labels. In this paper, we used the decision scheme described in MGOASVM [44]. In this scheme, the predicted subcellular location(s) of the $i$-th query protein are given by:

$$
\mathcal{M}^{*}\left(\mathbb{Q}_{t}\right)=\left\{\begin{array}{l}
\bigcup_{m=1}^{M}\left\{m: s_{m}\left(\mathbb{Q}_{t}\right)>0\right\}, \text { where } \exists s_{m}\left(\mathbb{Q}_{t}\right)>0 ; \\
\arg \max _{m=1}^{M} s_{m}\left(\mathbb{Q}_{t}\right), \text { otherwise. }
\end{array}\right.
$$

For ease of presentation, we refer to the two proposed predictors as mLASSO and mEN, respectively.

\section{Endnotes}

${ }^{1}$ http://www.proteinatlas.org/

${ }^{2}$ http://www.geneontology.org

${ }^{3} \mathrm{http} / /$ prosite.expasy.org/

${ }^{4}$ http://www.ebi.ac.uk/GOA

${ }^{5} \mathrm{http} / / /$ www.uniprot.org/ 
${ }^{6}$ Locative proteins are defined as follows. If a protein exists in two different subcellular locations, it will be counted as two locative proteins; if a protein coexists in three locations, then it will be counted as three locative proteins; and so forth.

${ }^{7}$ In our case, $N=3106$ for the human dataset.

${ }^{8}$ Specific weights $\left\{\beta_{s, m}\right\}_{s \in \mathcal{S}, m=\{1, \ldots, M\}}$ of each subcellular location for mLASSO and mEN can be found in Section S1 and S2, respectively, of the supplementary materials.

${ }^{9} \mathrm{Hum}$-mPLoc 2.0 [43] performs worse than iLoc-Hum, and only the $O L A$ is provided in [43]. Therefore, we do not report the performance of Hum-mPLoc 2.0 here.

${ }^{10}$ Note that because the number of novel reviewed human proteins that were added to Swiss-Prot after 2014 is too small to constitute a meaningful test set, we used some representative novel proteins to test $\mathrm{mEN}$ instead.

${ }^{11}$ The scores and weights for the essential GO terms for all of the 14 subcellular locations can be seen by inputing the query protein sequence to our SpaPredictor web-server.

${ }^{12}$ For ease of presentation, we omitted the bias in equations thereafter.

\section{Competing interests}

The authors declare that they have no competing interests.

\section{Authors' contributions}

WSB conceived the idea, designed the system, implemented programs and the web sever, carried out the analysis and participated in manuscript preparation. MMW designed the web application, carried out the analysis, reviewed the study and participated in manuscript preparation. KSY reviewed the study and participated in manuscript preparation. All authors read and approved the final manuscript.

\section{Acknowledgements}

This work was in part supported by the RGC of Hong Kong SAR Grant No. PolyU152117/14E.

\section{Author details}

${ }^{1}$ Department of Electronic and Information Engineering, The Hong Kong Polytechnic University, Hong Kong SAR, China. ${ }^{2}$ Department of Electrical Engineering, Princeton University, New Jersey, USA.

Received: 17 April 2015 Accepted: 27 January 2016

Published online: 24 February 2016

\section{References}

1. Hung MC, Link W. Protein localization in disease and therapy. J Cell Sci. 2011;124(Pt 20):3381-92.

2. Hayama A, Rai T, Sasaki S, Uchida S. Molecular mechanisms of Bartter syndrome caused by mutations in the BSND gene. Histochem Cell Biol. 2003;119(10):485-93.

3. Krutovskikh V, Mazzoleni G, Mironov N, Omori Y, Aguelon AM, Mesnil M, Berger F, Partensky C, Yamasaki H. Altered homologous and heterologous gap-junctional intercellular communication in primary human liver tumors associated with aberrant protein localization but not gene mutation of connexin 32. Int J Cancer. 1994;56:87-94.

4. Kaytor MD, Warren ST. Aberrant Protein Deposition and Neurological Disease. J Biol Chem. 1999;274:37507-10

5. Chen Y, Chen CF, Riley DJ, Allred DC, Chen PL, Hoff DV, Osborne CK, Lee WH. Aberrant Subcellular Localization of BRCA1 in Breast Cancer. Science. 1995;270:789-91.

6. Lee X, Keith JCJ, Stumm N, Moutsatsos I, McCoy JM, Crum CP, Genest D, Chin D, Ehrenfels C, Pijnenborg R, Assche FAV, Mi S. Downregulation of placental syncytin expression and abnormal protein localization in pre-eclampsia. Placenta. 2001;22:808-12

7. Campbell JB, Crocker J, Shenoi PM. S-100 protein localization in minor salivary gland tumours: an aid to diagnosis. J Laryngol Otol. 1988;102(10): 905-8.

8. Lubec G, Afjehi-Sadat L, Yang JW, John JP. Searching for hypothetical proteins: Theory and practice based upon original data and literature. Prog. Neurobiol. 2005;77:90-127.

9. Nakai K, Kanehisa M. Expert system for predicting protein localization sites in gram-negative bacteria. Proteins Struct Funct Genet. 1991;11(2):95-110.

10. Emanuelsson $\mathrm{O}$, Nielsen $\mathrm{H}$, Brunak S, von Heijne G. Predicting subcellular localization of proteins based on their $\mathrm{N}$-terminal amino acid sequence. J Mol Biol. 2000;300(4):1005-16.

11. Nielsen H, Engelbrecht J, Brunak S, von Heijne G. A neural network method for identification of prokaryotic and eukaryotic signal peptides and prediction of their cleavage sites. Int J Neural Sys. 1997:8:581-99.

12. Zhou GP, Doctor K. Subcellular location prediction of apoptosis proteins. Proteins Struct Funct Genet. 2003;50:44-8.

13. Fan GL, Li QZ. Predict mycobacterial proteins subcellular locations by incorporating pseudo-average chemical shift into the general form of Chou's pseudo amino acid composition. J Theor Biol. 2012;304:88-95.

14. Chou KC. Prediction of protein cellular attributes using pseudo amino acid composition. Proteins Struct Funct Genet. 2001:43:246-55.

15. Nakashima H, Nishikawa K. Discrimination of intracellular and extracellular proteins using amino acid composition and residue-pair frequencies. J Mol Biol. 1994;238:54-61.

16. Mak MW, Guo J, Kung SY. PairProSVM: Protein Subcellular Localization Based on Local Pairwise Profile Alignment and SVM. IEEE/ACM Trans Comput Biol Bioinformatics. 2008;5(3):416-22.

17. Mott R, Schultz J, Bork P, Ponting C. Predicting protein cellular localization using a domain projection method. Genome Res. 2002;12(8):1168-74.

18. Wan S, Mak MW. Machine learning for protein subcellular localization prediction, De Gruyter. Germany; 2015, p. 192. ISBN 978-1-5015-0150-0.

19. Lin WZ, Fang JA, Xiao X, Chou KC. iLoc-Animal: a multi-label learning classifier for predicting subcellular localization of animal proteins. Mol BioSyst. 2013;9(4):634-44.

20. Wan S, Mak MW, Kung SY. Protein subcellular localization prediction based on profile alignment and Gene Ontology. In: 2011 IEEE International Workshop on Machine Learning for Signal Processing (MLSP'11). New Jersey, USA: IEEE; 2011. p. 1-6.

21. Mei S. Multi-label multi-kernel transfer learning for human protein subcellular localization. PLOS ONE. 2012;7(6):e37716.

22. Wan S, Mak MW, Kung SY. Adaptive thresholding for multi-label SVM classification with application to protein subcellular localization prediction. In: 2013 IEEE International Conference on Acoustics, Speech, and Signal Processing (ICASSP'13). New Jersey, USA: IEEE; 2013. p. 3547-551.

23. Chou KC, Shen HB. Predicting eukaryotic protein subcellular location by fusing optimized evidence-theoretic K-nearest neighbor classifiers. J. Proteome Res. 2006;5:1888-97.

24. Wan S, Mak MW, Kung SY. Semantic similarity over gene ontology for multi-label protein subcellular localization. Engineering. 2013;5:68-72.

25. Chou KC, Cai YD. Prediction of protein subcellular locations by GO-FunD-PseAA predictor. Biochem Biophys Res Commun. 2004;320:1236-9.

26. Wan S, Mak MW, Kung SY. GOASVM: Protein subcellular localization prediction based on gene ontology annotation and SVM. In: 2012 IEEE International Conference on Acoustics, Speech, and Signal Processing (ICASSP'12). New Jersey, USA: IEEE; 2012. p. 2229-32.

27. Chou KC, Wu ZC, Xiao X. iLoc-Hum: using the accumulation-label scale to predict subcellular locations of human proteins with both single and multiple sites. Mol BioSyst. 2012;8:629-41.

28. Wan S, Mak MW, Zhang B, Wang Y, Kung SY. Ensemble random projection for multi-label classification with application to protein subcellular localization. In: 2014 IEEE International Conference on Acoustics, Speech and Signal Processing (ICASSP'14). New Jersey, USA; 2014. p 5999-6003.

29. Fyshe A, Liu Y, Szafron D, Greiner R, Lu P. Improving subcellular localization prediction using text classification and the gene ontology. Bioinformatics. 2008;24:2512-7.

30. Brady S, Shatkay H. EpiLoc: a (working) text-based system for predicting protein subcellular location. In: Pac. Symp. Biocomput. California, USA: Stanford University; 2008. p. 604-15. 
31. Nair R, Rost B. Sequence conserved for subcellular localization. Protein Sci. 2002;11:2836-47.

32. Lu Z, Szafron D, Greiner R, Lu P, Wishart DS, Poulin B, Anvik J, Macdonell C, Eisner R. Predicting subcellular localization of proteins using machine-learned classifiers. Bioinformatics. 2004;20(4):547-56.

33. Chi SM, Nam D. WegoLoc: accurate prediction of protein subcellular localization using weighted Gene Ontology terms. Bioinformatics. 2012;28(7):1028-1030. [http://bioinformatics.oxfordjournals.org/content/ 28/7/1028.short].

34. Wan S, Mak MW, Kung SY. GOASVM: A subcellular location predictor by incorporating term-frequency gene ontology into the general form of Chou's pseudo-amino acid composition. J Theor Biol. 2013;323:40-8.

35. Huang WL, Tung CW, Ho SW, Hwang SF, Ho SY. ProLoc-GO: Utilizing informative Gene Ontology terms for sequence-based prediction of protein subcellular localization. BMC Bioinformatics. 2008;9:80.

36. Murphy RF. communicating subcellular distributions. 77. 2010;7:686-92.

37. Millar AH, Carrie C, Pogson B, Whelan J. Exploring the function-location nexus: using multiple lines of evidence in defining the subcellular location of plant proteins. Plant Cell. 2009;21(6):1625-31.

38. Zhang S, Xia XF, Shen JC, Zhou Y, Sun Z. DBMLoc: A database of proteins with multiple subcellular localizations. BMC Bioinformatics. 2008;9:127.

39. Foster LJ, Hoog CLD, Zhang Y, Zhang Y, Xie X, Mootha VK, Mann M. A mammalian organelle map by protein correlation profiling. Cell. 2006;125: 187-99.

40. Rea S, James D. Moving GLUT4: the biogenesis and trafficking of GLUT4 storage vesicles. Diabetes. 1997;46:1667-77.

41. Russell R, Bergeron R, Shulman G, Young H. Translocation of myocardial GLUT-4 and increased glucose uptake through activation of AMPK by AICAR. Am J Physiol. 1997;277:H643-9.

42. Mueller JC, Andreoli C, Prokisch H, Meitinger T. Mechanisms for multiple intracellular localization of human mitochondrial proteins. Mitochondrion. 2004;3:315-25.

43. Shen HB, Chou KC. A top-down approach to enhance the power of predicting human protein subcellular localization: Hum-mPLoc 2.0. Ana Biochem. 2009;394(2):269-74.

44. Wan S, Mak MW, Kung SY. mGOASVM: Multi-label protein subcellular localization based on gene ontology and support vector machines. BMC Bioinformatics. 2012;13:290.

45. Wan S, Mak MW, Kung SY. HybridGO-Loc: Mining hybrid features on gene ontology for predicting subcellular localization of multi-location proteins. PLOS ONE. 2014;9(3):e89545.

46. Wan S, Mak MW, Kung SY. R3P-Loc: A compact multi-label predictor using ridge regression and random projection for protein subcellular localization. J Theor Biol. 2014;360:34-45.

47. Wan S, Mak MW, Kung SY. mPLR-Loc: An adaptive decision multi-label classifier based on penalized logistic regression for protein subcellular localization prediction. Anal Biochem. 2015;473:14-27.

48. He J, Gu H, Liu W. Imbalanced multi-modal multi-label learning for subcellular localization prediction of human proteins with both single and multiple sites. PLOS ONE. 2011;7(6):e37155

49. Li LQ, Zhang Y, Zou LY, Li CQ, Yu B, Zheng XQ, Zhou Y. An ensemble classifier for eukaryotic protein subcellular location prediction using Gene Ontology categories and amino acid hydrophobicity. PLOS ONE. 2012;7: e31057.

50. Wan S, Mak MW, Zhang B, Wang Y, Kung SY. An ensemble classifier with random projection for predicting multi-label protein subcellular localization. In: 2013 IEEE International Conference on Bioinformatics and Biomedicine (BIBM). New Jersey, USA: IEEE; 2013. p. 35-42.

51. Briesemeister S, Rahnenführer J, Kohlbacher O. YLoc-an interpretable web server for predicting subcellular localization. Nucleic Acids Res. 2010;38(Suppl 2):W497-502.

52. Lu Z, Hunter L. GO molecular function terms are predictive of subcellular localization. In: Proc. of Pac. Symp. Biocomput (PSB'05). California, USA: Stanford University; 2005. p. 151-61.

53. Altschul SF, Madden TL, Schaffer AA, Zhang J, Zhang Z, Miller W, Lipman DJ. Gapped BLAST and PSI-BLAST: A new generation of protein database search programs. Nucleic Acids Res. 1997;25:3389-402.

54. Briesemeister S, Blum T, Brady S, Lam Y, Kohlbacher O, Shatkay H. SherLoc2: A high-accuracy hybrid method for predicting subcellular localization of proteins. J Proteome Res. 2009;8:5363-6.

55. Chou KC. Some remarks on predicting multi-label attributes in molecular biosystems. Mol BioSyst. 2013;9:1092-100.
56. Wang X, Li GZ. A multi-label predictor for identifying the subcellular locations of singleplex and multiplex eukaryotic proteins. PLoS ONE. 2012;7(5):e36317.

57. Chou KC, Shen HB. Cell-PLoc: A package of web-servers for predicting subcellular localization of proteins in various organisms. Nat Protoc. 2008;3:153-62.

58. Wu ZC, Xiao X, Chou KC. iLoc-Plant: A multi-label classifier for predicting the subcellular localization of plant proteins with both single and multiple sites. Mol BioSyst. 2011;7:3287-97.

59. Dembczynski K, Waegeman W, Cheng W, Hullermeier E. On label dependence and loss minimization in multi-label classification. Mach Learn. 2012;88(1-2):5-45.

60. Gao W, Zhou ZH. On the consistency of multi-label learning. In: Proceedings of the 24th Annual Conference on Learning Theory. Massachusetts, USA: Microtome Publishing; 2011. p. 341-58.

61. Clark WT, Radivojac P. Information-theoretic evaluation of predicted ontological annotations. Bioinformatics. 2013;29(13):i53-61.

62. Verspoor K, Cohn J, Mniszewski S, Joslyn C. A categorization approach to automated ontological function annotation. Protein Sci. 2006;15(6):1544-9.

63. Tsoumakas G, Katakis I, Vlahavas I. Random k-labelsets for multilabel classification. IEEE Trans Knowl Data Eng. 2011;23(7):1079-89.

64. Manning CD, Raghavan $P$, Schütze $H$. Introduction to information retrieval Volume 1. Cambridge: Cambridge university press; 2008.

65. Xiao X, Wu ZC, Chou KC. iLoc-Virus: A multi-label learning classifier for identifying the subcellular localization of virus proteins with both single and multiple sites. J Theor Biol. 2011;284:42-51.

66. Hastie T, Tibshirani R, Friedman J. The element of statistical learning. Berlin, Germany: Springer-Verlag; 2001.

67. Zou H, Hastie T. Regularization and variable selection via the elastic net J R Stat Soc Ser B Stat Methodol. 2005;67(2):301-20.

68. Chou KC, Wu ZC, Xiao X. iLoc-Euk: A multi-label classifier for predicting the subcellular localization of singleplex and multiplex eukaryotic proteins. PLoS ONE. 2011;6(3):e18258.

69. Wan S, Mak MW, Kung SY. mLASSO-Hum: A LASSO-based interpretable human-protein subcellular localization predictor. J Theor Biol. 2015;382: 223-34.

70. Nakai K. Protein sorting signals and prediction of subcellular localization. Adv Protein Chem. 2000;54:277-344.

71. Wan S, Mak MW, Kung SY. Mem-mEN: Predicting multi-functional types of membrane proteins by interpretable elastic nets. IEEE/ACM Trans Comput Biol Bioinformatics. 2015. doi:10.1109/TCBB.2015.2474407.

72. Tibshirani R. Regression shrinkage and selection via the Lasso. J R Stat Soc Ser B Methodol. 1996267-88.

73. Zhang B, Li H, Riggins RB, Zhan M, Xuan J, Zhang Z, Hoffman EP, Clarke R, Wang Y. Differential dependency network analysis to identify condition-specific topological changes in biological networks. Bioinformatics. 2009;25(4):526-32.

74. Lu Y, Zhou Y, Qu W, Deng M, Zhang C. A Lasso regression model for the construction of microRNA-target regulatory networks. Bioinformatics. 2011;27(17):2406-13

75. de Maturana EL, Ye Y, Calle ML, Rothman N, Urrea V, et al. Application of multi-SNP approaches Bayesian LASSO and AUC-RF to detect main effects of inflammatory-gene variants associated with bladder cancer risk. PLOS ONE. 2013;8(12):e83745.

76. Vasilevski A, Giorgi FM, Bertinetti L, Usadel B. LASSO modeling of the Arabidopsis thaliana seed/seedling transcriptome: a model case for detection of novel mucilage and pectin metabolism genes. Mol BioSyst. 2012;8(10):2566-74.

77. He D, Wang Z, Parida L. Data-driven encoding for quantitative genetic trait prediction. BMC Bioinformatics. 2015;16(Suppl 1):S10.

78. Marafino BJ, Boscardin WJ, Dudley RA. Efficient and sparse feature selection for biomedical text classification via the elastic net: Application to ICU risk stratification from nursing notes. J Biomed Inf. 2015;54:114-20.

79. Ayers KL, Cordell HJ. SNP Selection in genome-wide and candidate gene tudies via penalized logistic regression. Genet Epidemiol. 2010;34(8):879-91.

80. Efron B, Hastie T, Johnstone I, Tibshirani R. Least angle regression. Ann Stat. 2004:32(2):407-99.

81. Sjöstrand K, Clemmensen LH, Larsen R, Ersbøll B. SpaSM: A Matlab Toolbox for Sparse Statistical Modeling. J Stat Softw. Accepted for publication. 2004. http://www.imm.dtu.dk/projects/spasm/references/ spasm.pdf. 